\title{
Testing of quasi-elastic neutrino charged-current and two-body meson exchange current models with the MiniBooNE neutrino data and analysis of these processes at energies available at the NOvA experiment
}

\author{
A. V. Butkevich ${ }^{1}$ and S. V. Luchuk ${ }^{1,2}$ \\ ${ }^{1}$ Institute for Nuclear Research, Russian Academy of Sciences, Moscow 117312, Russia \\ ${ }^{2}$ Moscow Institute of Physics and Technology, Dolgoprudny 141701, Russia
}

(Received 10 January 2019; published 1 May 2019)

\begin{abstract}
The charged-current quasi-elastic scattering of muon neutrinos on a carbon target is analyzed using the relativistic distorted-wave impulse approximation (RDWIA), taking into account the contribution of the two-particle and two-hole meson exchange current (MEC) to the weak response functions. A fit the RDWIA + MEC model to the MiniBooNE neutrino data is performed, and the best-fit value of nucleon axial mass $M_{A} \approx 1.20 \mathrm{GeV}$ is obtained. We also extract the values of the axial form factor $F_{A}\left(Q^{2}\right)$ as a function of the squared momentum transfer $Q^{2}$ from the measured $d \sigma / d Q^{2}$ cross section. The fluxintegrated charged-current quasi-elastic-like differential cross sections for neutrino scattering at energies of the NOvA experiment are estimated within the RDWIA + MEC approach.
\end{abstract}

DOI: 10.1103/PhysRevD.99.093001

\section{INTRODUCTION}

The high-intensity muon-(anti)neutrino beams used in long-baseline neutrino oscillation experiments are peaked in the energy range from a few hundreds of mega-electronvolts to several giga-electron-volts. In this energy regime, the dominant contribution to neutrino-nucleus scattering comes from the charged-current quasi-elastic (CCQE) interaction, two-body meson exchange current (MEC), resonance production, and deep inelastic processes. To determine values of neutrino oscillation parameters, the probabilities of $\nu_{\mu}$ disappearance and $\nu_{e}$ appearance vs neutrino energy are extracted in these experiments. The accuracy of these measurements depends explicitly on how well we are able to evaluate the energy of the incoming neutrino. This energy can be estimated from the lepton and hadron energies visible in the final state after the neutrino has interacted.

In the genuine CCQE neutrino scattering on nuclei, the incoming neutrino energy can be reconstructed using outgoing lepton kinematics and average nucleon binding energy, assuming the target nucleon to be at rest inside the nucleus. The measurement of muon momentum and angle allows the estimation of the neutrino energy $\varepsilon_{\nu}$ and the squared four-momentum transfer $Q^{2}$. This reconstruction

Published by the American Physical Society under the terms of the Creative Commons Attribution 4.0 International license. Further distribution of this work must maintain attribution to the author(s) and the published article's title, journal citation, and DOI. Funded by SCOAP . method (kinematic method) would work well if the true nature of events were indeed a CCQE process. Nuclear effects, the final-state interaction (FSI) between of the outgoing particles and residual nucleua as well as interactions which are not distinguishable from CCQE in the final state lead to bias or smear the reconstructed neutrino energy. Therefore, a good understanding of these effects is critical.

To model the CCQE scattering from a nuclei, most event generators are based on the relativistic Fermi gas model (RFGM) [1]. In this model, the nucleus is described as a system of quasi-free nucleons with a flat momentum distribution up to the Fermi momentum $p_{F}$ and nuclear binding energy $\epsilon_{b}$. With the assumption of the conserved vector current, the only parameter of the weak current which is not well constrained by electron scattering data is the axial nucleon form factor $F_{A}\left(Q^{2}\right)$. In most analyses of the CCQE interaction, the dipole parametrization of $F_{A}\left(Q^{2}\right)$ with one parameter, the axial mass $M_{A}$, is used. Note that dipole parametrization has no strict theoretical basis, and the choice of this parametrization is made by analogy with electromagnetic form factors.

The value of $M_{A}$ is obtained from a fit to observed $Q^{2}$ distribution of events, differential, and total (anti)neutrino CCQE cross sections. Results from global analysis of neutrino-deuterium scattering experiments are very widely spread, and the formal averaging of $M_{A}$ values was done in Ref. [2]: $M_{A}=1.026 \pm 0.021 \mathrm{GeV}$. This result is also known as the world-averaged value of the axial mass. The NOMAD experiment has reported result on neutrino CCQE scattering on carbon: $M_{A}=1.05 \pm 0.02 \pm$ $0.06 \mathrm{GeV}$ [3]. The MINERvA experiment [4,5] has shown 
good agreement within the RFGM with $M_{A} \approx 1 \mathrm{GeV}$ but requires an enhancement to the transverse response function. A recent reanalysis of the MINERvA flux [6] resulted in the increases of the normalization of previous cross sections $[7,8]$ and invalidated conclusions from Refs. $[4,5]$.

On the other hand, the differential cross sections measured by the MiniBooNE Collaboration [9-11] can be described within the RFGM only with a large value of $M_{A}=1.35 \pm 0.017 \mathrm{GeV}$. The absolute values of the differential and total cross sections are about 30\% larger compared to NOMAD results. Large values of axial mass $M_{A} \approx 1.1-1.3 \mathrm{GeV}$ have also been obtained in other experiments using heavy nuclear targets [12-15].

These results have stimulated many theoretical studies trying to explain the apparent discrepancy between the data and theoretical predictions and present a considerable challenge to neutrino oscillation experiments. A wide variety of models has been proposed to describe CCQElike cross sections, identified experimentally as processes in which only a final charged lepton with multinucleon excitations is detected, but the pion absorption contribution is subtracted. The data without subtracting any intrinsic background are called $\mathrm{CC} 0 \pi$. A review of the available CCQE-like cross section models can be found in Refs. [16-19].

Based on the results from different groups, it is shown that CCQE-like data are really a combination of the genuine quasi-elastic (QE) and of the two-particle and two-hole meson exchange current $(2 p-2 h$ MEC) contributions to weak response functions. Such excitations are induced by two-body currents; hence, they go beyond the usual impulse approximation (IA) scheme, in which the probe interacts with only a single nucleon and corresponds to the $1 p-1 h$ excitations. So, the $2 p-2 h$ MEC contributions to the electroweak response functions are corrections to the $1 p-1 h$ leading channel of the $\mathrm{QE}$ lepton scattering on nuclei. To describe the genuine QE, a model should, in principle, include the nuclear mean-field and nucleon-nucleon $(N N)$ short- and long-range correlations in the ground state as well as final-state interaction of the outgoing nucleon with the residual nucleus. Because the virtual photon (boson) couples to only one member of the $N N$ pair, it is a one-body current process, even though it leads to the emission of two nucleons. More sophisticated descriptions of the genuine CCQE interaction than the RFGM provides are available from a number authors [20-31]. Note that there exist some differences already at the level of the genuine quasi-elastic scattering.

The transverse enhancement effective model to account for MEC effects has been proposed in Ref. [32]. In this model, the magnetic form factor for nucleon bound in carbon is modified to describe the enhancement in the electron-carbon QE cross section. An enhancement of the axial nucleon mass in the nonrelativistic continuum random phase approximation has been regarded in Ref. [33].
The contribution of the $n p-n h$ channel is also taken into account through a phenomenological approach in Ref. [34].

The most complete theoretical calculations of $2 p-2 h$ cross sections are performed by different groups [35-43]. In Refs. [35-38], the models start from a local Fermi gas picture of the nucleus and take into account long-range random phase approximation (RPA) corrections but ignore the shell structure of nucleus and FSI effects. In the $2 p-2 h$ sector, both models use the Fermi gas approximation. The short-range $N N$ correlations are included by considering an additional two-body correlation current. As a result, the $N N$ correlations and $N N$ correlations-MEC interference naturally appear (RPA-MEC approach).

In the SuperScaling Approach (SuSA) [30,39-42], a superscaling analysis of the electron scattering result is used to calculate neutrino cross sections. The effects of the short-range $N N$ correlations in the $1 p-1 h$ sector are effectively included via the superscaling function. In Ref. [41], the SuSAv2 model is combined with MECs in the $2 p-2 h$ sector by using accurate parametrizations of electroweak MEC response functions [39,40], calculated within the RFGM (SuSAv2-MEC approach). The $N N$ correlations and $N N$ correlations-MEC interference are absent in the $2 p-2 h$ MEC contributions.

Another approach which goes beyond the impulse approximation was developed in Ref. [44]. In this work, a joint calculation of the CCQE and $2 p-2 h$ contributions to the lepton scattering cross sections on carbon, using relativistic distorted-wave impulse approximation (RDWIA) for quasi-elastic response functions in the electroweak sector (RDWIA + MEC approach), was performed. The RDWIA, which takes into account the nuclear shell structure and final-state interaction effects, was developed to describe of the QE electron-nucleus scattering [45-47]. Results of the data analysis for data for ${ }^{12} \mathrm{C}\left(e, e^{\prime} p\right)$ based on upon the RDWIA can be found in Refs. [24,47], which show that the nucleon momentum distributions are described well by mean-field calculations. In our approach $[23,24]$, the effects of the short-range $N N$ correlations, leading to the appearance of a high-momentum and highenergy distribution in the target, are estimated.

We explicitly added the MEC contributions (without the $N N$ correlations-MEC interference) to the genuine QE interaction, as in the SuSA-MEC approach [41]. The functional forms employed for the parametrizations of the MEC transverse electromagnetic vector response and for the axial and vector components of the weak response were detailed in Refs. [41,42]. These parametrizations allow us to quickly calculate the MEC contributions for the lepton scattering on carbon. Additionally, for development of the new generation of neutrino generators, reasonably successful models which need only short computing time may be a useful bridge. On a long timescale, one has to aim for $a b$ initio calculations. The RDWIA + MEC approach was successfully tested against ${ }^{12} \mathrm{C}\left(e, e^{\prime}\right)$ data [44]. 
Although theoretical calculations of the CCQE-like neutrino-nucleus cross sections have been performed by many groups using different approaches, at this moment, there is no progress in a quantitative description of the data, and it is not clear which models fit the global data best. For example, the global fit performed in Ref. [48] shows very poor results. One of the goals of this paper is to fit the RDWIA + MEC model to the MiniBooNE data [9] for neutrino scattering off carbon. Within this approach, we extract the value of the axial mass from measured fluxintegrated $d \sigma / Q^{2}$ and $d^{2} \sigma / d T d \cos \theta$ ( $T$ and $\theta$ are, correspondingly, kinetic energy and muon scattering angle) differential cross sections. In addition, we determine the values of the axial form factor $F_{A}\left(Q^{2}\right)$ as a function of $Q^{2}$, using the method described in Ref. [49]. Previously, our constraint on the $M_{A} \approx 1.37 \mathrm{GeV}$ was obtained within the RDWIA [49,50]. This work improves the previous situation by including $2 p-2 h$ MEC contributions. A second topic addressed in this paper is calculations of the CCQE-like flux-integrated differential and double-differential cross sections at energies of the NOvA experiment [51,52]. We evaluated these cross sections within the RDWIA + MEC approach with the value of $M_{A}$ extracted from the MiniBooNE data.

This article is organized as follows. In Sec. II, we briefly present the RDWIA + MEC model and the procedure which allows the determination of values of the axial form factor from the $d \sigma / d Q^{2}$ differential cross section. Section III presents results of this model to the MiniBooNE data, extraction of the $F_{A}\left(Q^{2}\right)$, and calculations of the flux-integrated differential cross sections for the NOvA experiment. Our conclusions are summarized in Sec. IV.

\section{FORMALISM OF QUASI-ELASTIC SCATTERING, RDWIA, $2 p-2 h$ MEC RESPONSES, AND FLUX-INTEGRATED CROSS SECTIONS}

We consider neutrino charged-current $\mathrm{QE}$ inclusive

$$
\nu_{\mu}\left(k_{i}\right)+A\left(p_{A}\right) \rightarrow \mu\left(k_{f}\right)+X
$$

scattering off nuclei in the one-W boson exchange approximation. Here, $k_{i}=\left(\varepsilon_{i}, \boldsymbol{k}_{i}\right)$ and $k_{f}=\left(\varepsilon_{f}, \boldsymbol{k}_{f}\right)$ are the initial and final lepton momenta, $p_{A}=\left(\varepsilon_{A}, \boldsymbol{p}_{A}\right)$ is the initial target momenta, $q=(\omega, \boldsymbol{q})$ is the momentum transfer carried by the virtual $\mathrm{W}$ boson, and $Q^{2}=-q^{2}=$ $\boldsymbol{q}^{2}-\omega^{2}$ is the W-boson virtuality.

\section{A. CCQE-like quasi-elastic lepton-nucleus cross sections}

In the inclusive reactions (1), only the outgoing lepton is detected, and the differential cross sections can be written as

$$
\frac{d^{3} \sigma}{d \varepsilon_{f} d \Omega_{f}}=\frac{1}{(2 \pi)^{2}} \frac{\left|\boldsymbol{k}_{f}\right|}{\varepsilon_{i}} \frac{G^{2} \cos ^{2} \theta_{c}}{2} L_{\mu \nu} W^{\mu \nu},
$$

where $\Omega_{f}=(\theta, \phi)$ is the solid angle for the muon momentum, $G \simeq 1.16639 \times 10^{-11} \mathrm{MeV}^{-2}$ is the Fermi constant, $\theta_{C}$ is the Cabbibo angle $\left(\cos \theta_{C} \approx 0.9749\right), L_{\mu \nu}$ is the lepton tensor, and $W^{\mu \nu}$ are the charged-current (CC) nuclear tensor. In terms of response functions, the cross sections reduce to

$$
\begin{aligned}
\frac{d^{3} \sigma}{d \varepsilon_{f} d \Omega_{f}}= & \frac{G^{2} \cos ^{2} \theta_{c}}{(2 \pi)^{2}} \varepsilon_{f}\left|\boldsymbol{k}_{f}\right|\left(v_{0} R_{0}+v_{T} R_{T}+v_{z z} R_{z z}\right. \\
& \left.-v_{0 z} R_{0 z}-h v_{x y} R_{x y}\right),
\end{aligned}
$$

where the coupling coefficients $v_{k}$, the expressions of which are given in Ref. [23] are kinematic factors depending on the lepton's kinematics. The response functions are given in terms of components of the hadronic tensors

$$
\begin{gathered}
R_{0}=W^{00}, \\
R_{T}=W^{x x}+W^{y y}, \\
R_{0 z}=W^{0 z}+W^{z 0}, \\
R_{z z}=W^{z z}, \\
R_{x y}=i\left(W^{x y}-W^{y x}\right)
\end{gathered}
$$

and depend on the variables $\left(Q^{2}, \omega\right)$ or $(|q|, \omega)$.

All the nuclear structure information and final-state interaction effects are contained in the weak CC nuclear tensor. It is given by the bilinear products of the transition matrix elements of the nuclear CC operator $J_{\mu}^{c c}$ between the initial nucleus state $|A\rangle$ and the final state $\left|X_{f}\right\rangle$ as

$$
W_{\mu \nu}=\sum_{f}\left\langle X_{f}\left|J_{\mu}^{(c c)}\right| A\right\rangle\left\langle A\left|J_{\nu}^{(c c) \dagger}\right| X_{f}\right\rangle
$$

where the sum is taken over undetected states $X_{f}$. This equation includes all possible final states. Thus, the hadron tensor can be expanded as the sum of the $1 p-1 h$ and $2 p-2 h$, plus additional channels,

$$
W^{\mu \nu}=W_{1 p 1 h}^{\mu \nu}+W_{2 p 2 h}^{\mu \nu}+\cdots,
$$

where the $1 p-1 h$ channel gives the CCQE response functions and the $2 p-2 h$ hadronic tensor determines the $2 p-2 h$ MEC response functions. Thus, the functions $R_{i}$ Eqs. (4a)-(4e) can be written as a sum of the CCQE $\left(R_{i, \mathrm{QE}}\right)$ and $\mathrm{MEC}\left(R_{i, \mathrm{MEC}}\right)$ response functions

$$
R_{i}=R_{i, \mathrm{QE}}+R_{i, \mathrm{MEC}} .
$$




\section{B. RDWIA model}

We describe genuine CCQE neutrino-nuclear scattering in the impulse approximation, assuming that the incoming neutrino interacts with only one nucleon, which is subsequently emitted, while the remaining (A-1) nucleons in the target are spectators. The nuclear current is written as the sum of single-nucleon currents.

The single-nucleon charged current has $V-A$ structure $J^{\mu}=J_{V}^{\mu}+J_{A}^{\mu}$. For a free-nucleon vertex function $\Gamma^{\mu}=$ $\Gamma_{V}^{\mu}+\Gamma_{A}^{\mu}$, we use vector current vertex function $\Gamma_{V}^{\mu}=$ $F_{V}\left(Q^{2}\right) \gamma^{\mu}+i F_{M}\left(Q^{2}\right) \sigma^{\mu \nu} q_{\nu} / 2 m$, where $\sigma^{\mu \nu}=i\left[\gamma^{\mu}, \gamma^{\nu}\right] / 2$ and $F_{V}$ and $F_{M}$ are the weak vector form factors. They are related to the corresponding electromagnetic ones for the proton and neutron by the hypothesis of the conserved vector current. We use the approximation of Ref. [53] for the vector nucleon form factors. The differences between these vector form factors and newer parametrizations are not significant in the range of $Q^{2}<3(\mathrm{GeV} / c)^{2}$, which gives the main contribution to the $\mathrm{CCQE}$-like cross sections. Because the bound nucleons are off shell, we employ the de Forest prescription [54] and use the Coulomb gauge for the off-shell vector current vertex $\Gamma_{V}^{\mu}$.

The axial current vertex function can be written in terms of the axial $F_{A}\left(Q^{2}\right)$ and pseudoscalar $F_{P}$ form factors

$$
\Gamma_{A}^{\mu}=F_{A}\left(Q^{2}\right) \gamma^{\mu} \gamma_{5}+F_{P}\left(Q^{2}\right) q^{\mu} \gamma_{5} .
$$

The pseudoscalar form factor $F_{P}\left(Q^{2}\right)$ is dominated by the pion pole and is given in terms of the Goldberger-Treiman relation near $Q^{2} \approx 0$ if a partially conserved axial current is assumed. We assume that the similar relation is valid for high $Q^{2}$ as well. These form factors are parametrized using a dipole approximation,

$F_{A}\left(Q^{2}\right)=\frac{F_{A}(0)}{\left(1+Q^{2} / M_{A}^{2}\right)^{2}}, \quad F_{P}\left(Q^{2}\right)=F_{A}\left(Q^{2}\right) F_{P}^{\prime}\left(Q^{2}\right)$,

where $F_{P}^{\prime}=2 m^{2} /\left(m_{\pi}^{2}+Q^{2}\right), F_{A}(0)=1.2724, M_{A}$ is the axial mass, and $m_{\pi}$ is the pion mass. Then, the axial current vertex function can be written in the form

$$
\Gamma_{A}^{\mu}=F_{A}\left(Q^{2}\right)\left[\gamma^{\mu} \gamma_{5}+F_{P}^{\prime}\left(Q^{2}\right) q^{\mu} \gamma_{5}\right],
$$

and the axial-vector current can be factorized as

$$
J_{A}=F_{A}\left(Q^{2}\right) J_{A}^{\prime}\left(Q^{2}\right),
$$

where $J_{A}^{\prime}=\gamma^{\mu} \gamma_{5}+F_{P}^{\prime}\left(Q^{2}\right) q^{\mu} \gamma_{5}$

In the RDWIA, the relativistic wave functions of the bound nucleon states are calculated in the independent particle shall model (IPSM) as the self-consistent solutions of a Dirac equation, derived within a relativistic mean-field approach, from a Lagrangian containing $\sigma, \omega$, and $\rho$ mesons (the $\sigma-\omega$ model) $[55,56]$. According to the JLab data [47,57], the occupancy $S(\alpha)$ of the independent particle shell model orbitals of ${ }^{12} \mathrm{C}$ are equal, $S\left(1 p_{1 / 2}\right)=84 \%, S\left(1 s_{1 / 2}\right)=100 \%$, and equal an average $89 \%$. In this work, we assume that the missing strength $(11 \%)$ can be attributed to the short-range $N N$ correlations in the ground state, leading to the appearance of the high-momentum and high-energy component in the nucleon distribution in the target. In the RDWIA, the final-state interaction effects for the outgoing nucleon are taken into account. The distorted-wave function of the knocked-out nucleon is evaluated as a solution of a Dirac equation containing a phenomenological relativistic optical potential. The EDAD1 parametrization [58] of the relativistic optical potential for carbon was used in this work. We calculated the inclusive cross sections with the EDAD1 relativistic optical potential in which only the real part was included. The data sets from the electron scattering experiments $[47,57]$ demonstrate the preference for this optical potential over other parametrizations. This was concluded based on the quality of the fits and more consistent nature of the extracted values of the occupancy $S(\alpha)$ of the IPSM orbitals.

The cross sections with the FSI effects in the presence of the short-range $N N$ correlations were calculated by using the method proposed in Ref. [23] with the nucleon high-momentum distribution from Ref. [59] that was renormalized to value of $11 \%$. In this approach, the contribution of the $N N$-correlated pairs is evaluated in impulse approximation; i.e., the virtual $\mathrm{W}$ boson couples to only one member of the $N N$ pair. It is a one-body process that leads to the emission of two nucleons $(2 p-2 h$ excitation).

\section{C. $2 p-2 h$ excitation}

In the present work, we evaluate the weak MEC response functions $R_{i, \mathrm{MEC}}$ of neutrino scattering on carbon, using accurate parametrizations of the exact MEC calculation [40]. To evaluate the $2 p-2 h$ hadronic tensor $W_{2 p 2 h}^{\mu \nu}$, in Ref. [40], the RFGM was chosen to describe the nuclear ground state. The short-range $N N$ correlations and FSI effects were not considered in this approach. The elementary hadronic tensor is given by the bilinear product of the matrix elements of the two-body weak (containing vector and axial components) MEC. Only one-pion exchange is included.

The two-body current operator is obtained from the pion production amplitudes for the nucleon while coupling a second nucleon to the emitted pion. The resulting MEC operator can be written as a sum of seagull, pion-in-flight, pion-pole, and Delta-pole operators. The $\Delta$ peak is the main contribution to the pion production cross section, and the MEC peak is located in the "dip" region between the QE and Delta peaks. 
The functional forms employed for these parametrizations as functions of $(\omega,|\boldsymbol{q}|)$ are valid in the range of momentum transfer $|\boldsymbol{q}|=200-2000 \mathrm{MeV}$. The expressions for the fitting parameters are described in detail in Refs. [41,42,60]. Results of lepton-nucleus cross sections obtained using these MEC parametrizations were successfully tested against the experimental world data for ${ }^{12} \mathrm{C}[42,44,61]$.

\section{Flux-integrated cross sections and the method for extraction of $F_{A}\left(Q^{2}\right)$ from $d \sigma / d Q^{2}$ distribution}

The inclusive weak hadronic tensor is bilinear in the transition matrix elements of the nuclear weak current operators $W_{\mu \nu}=\left\langle J_{\mu} J_{\nu}^{\dagger}\right\rangle$, where the angle brackets denote products of matrix elements appropriately averaged over initial states and summed over final states. By using Eq. (11), the axial-vector current can be written as $J=J_{V}+F_{A} J_{A}^{\prime}$. The expressions for the inclusive CCQE cross sections in terms of vector $\sigma^{V}$, axial $\sigma^{A}$, and vectoraxial $\sigma^{V A}$ cross sections are then given by [49]

$$
\begin{aligned}
\frac{d \sigma^{\nu}}{d Q^{2}}\left(Q^{2}, \varepsilon_{i}\right)= & \sigma^{V}\left(Q^{2}, \varepsilon_{i}\right)+F_{A}^{2}\left(Q^{2}\right) \sigma^{A}\left(Q^{2}, \varepsilon_{i}\right) \\
& +h F_{A}\left(Q^{2}\right) \sigma^{V A}\left(Q^{2}, \varepsilon_{i}\right)
\end{aligned}
$$

$$
\begin{aligned}
\frac{d^{2} \sigma^{\nu}}{d T d \cos \theta}\left(T, \cos \theta, \varepsilon_{i}\right)= & \sigma^{V}\left(T, \cos \theta, \varepsilon_{i}\right) \\
& +F_{A}^{2}\left(Q^{2}\right) \sigma^{A}\left(T, \cos \theta, \varepsilon_{i}\right) \\
& +h F_{A}\left(Q^{2}\right) \sigma^{V A}\left(T, \cos \theta, \varepsilon_{i}\right),
\end{aligned}
$$

where $\sigma^{V}$ is the cross section $d \sigma / d Q^{2}\left(d^{2} \sigma / d T d \cos \theta\right)$ calculated with $F_{A}=0$ and $\sigma^{A}$ is the cross section $d \sigma / d Q^{2}\left(d^{2} \sigma / d T d \cos \theta\right)$ calculated with $F_{V}=F_{M}=0$, $F_{A}=1$. The vector-axial cross section $\sigma^{V A}$, arising from the interference between the vector and axial currents, can be written as

$$
\sigma^{V A}=h\left[\sigma\left(F_{A}=1\right)-\sigma^{V}-\sigma^{A}\right],
$$

where $\sigma\left(F_{A}=1\right)$ is the $d \sigma / d Q^{2}\left(d^{2} \sigma / d T d \cos \theta\right)$ cross section, calculated with $F_{A}\left(Q^{2}\right)=1$.

The flux-integrated cross section can be written as a sum of the flux-integrated $\mathrm{QE}$ and $2 p-2 h$ MEC contributions

$\left\langle\frac{d \sigma}{d Q^{2}}\left(Q^{2}\right)\right\rangle=\left\langle\frac{d \sigma_{\mathrm{QE}}}{d Q^{2}}\left(Q^{2}\right)\right\rangle+\left\langle\frac{d \sigma_{\mathrm{MEC}}}{d Q^{2}}\left(Q^{2}\right)\right\rangle$,

where

$$
\left\langle\frac{d \sigma_{j}}{d Q^{2}}\left(Q^{2}\right)\right\rangle=\int D_{\nu}\left(\varepsilon_{i}\right) \frac{d \sigma_{j}}{d Q^{2}}\left(Q^{2}, \varepsilon_{i}\right) d \varepsilon_{i},
$$

$D_{\nu}$ is a unit-normalized neutrino flux, and $j=$ QE, MEC.

The value of $F_{A}\left(Q^{2}\right)$ as a function of $Q^{2}$ can be extracted as the solution to the equation

$$
\left\langle\frac{d \sigma_{\mathrm{QE}}}{d Q^{2}}\left(Q^{2}\right)\right\rangle=\left\langle\frac{d \sigma}{d Q^{2}}\left(Q^{2}\right)\right\rangle-\left\langle\frac{d \sigma_{\mathrm{MEC}}}{d Q^{2}}\left(Q^{2}\right)\right\rangle,
$$

using the neutrino CCQE-like scattering data for $\left\langle d \sigma^{\nu} / d Q^{2}\right\rangle$ and

$$
\begin{aligned}
\left\langle\frac{d \sigma_{\mathrm{QE}}}{d Q^{2}}\left(Q^{2}\right)\right\rangle= & \left\langle\sigma^{V}\left(Q^{2}\right)\right\rangle+F_{A}^{2}\left(Q^{2}\right)\left\langle\sigma^{A}\left(Q^{2}\right)\right\rangle \\
& +h F_{A}\left(Q^{2}\right)\left\langle\sigma^{V A}\left(Q^{2}\right)\right\rangle,
\end{aligned}
$$

where $\left\langle\sigma^{j}\left(Q^{2}\right)\right\rangle$ are the flux-integrated vector, axial, and vector-axial $(j=V, A, V A)$ cross sections. Note that the values of the axial form factor extracted from the CCQElike cross sections are model dependent and implicitly include the uncertainties in the $F_{V}, F_{M}, \nu_{\mu}$-flux, and $2 p-2 h$ MEC contributions.

\section{RESULTS AND ANALYSIS}

Our main interest is to show the capability of the present model, RDWIA + MEC, of successfully describing the MiniBooNE neutrino scattering data and calculating within this approach the CCQE-like flux-integrated cross sections at energies available at the NOvA experiment.

\section{A. Fit of the RDWIA + MEC model to the neutrino MiniBooNE data}

The MiniBooNE neutrino data set [9], obtained in a kinematic range that significantly overlaps with the range available to the NOvA experiment, is used in the CCQElike fit. These data have been released as flux-integrated double-differential cross section $d^{2} \sigma / d T d \cos \theta$ and as differential cross section $d \sigma / d Q^{2}$ in the range $0<Q^{2}<$ $2(\mathrm{GeV} / c)^{2}$. The single-differential cross section results are given in terms of $Q_{\mathrm{QE}}^{2}$, the four-momentum transfer calculated from the outgoing muon energy and angle. The data release included the diagonal elements of the shape-only covariant matrix for each bin, and correlations between bins were not presented. The normalization uncertainly was given as $10.7 \%$. In our analysis, we use the CCQE corrected sample with purity $77 \%$. In the MiniBooNE antineutrino data set [11], the correction algorithm for the antineutrino data is more complicated than for neutrino mode sample, due to the relatively high $\nu_{\mu}$ contamination in the $\bar{\nu}_{\mu}$ beam. There is also a large $\mathrm{CC} 1 \pi^{-}$ background in the $\bar{\nu}_{\mu}$ CCQE sample, as most of the $\pi^{-}$are absorbed. As a result of the two large backgrounds in the 


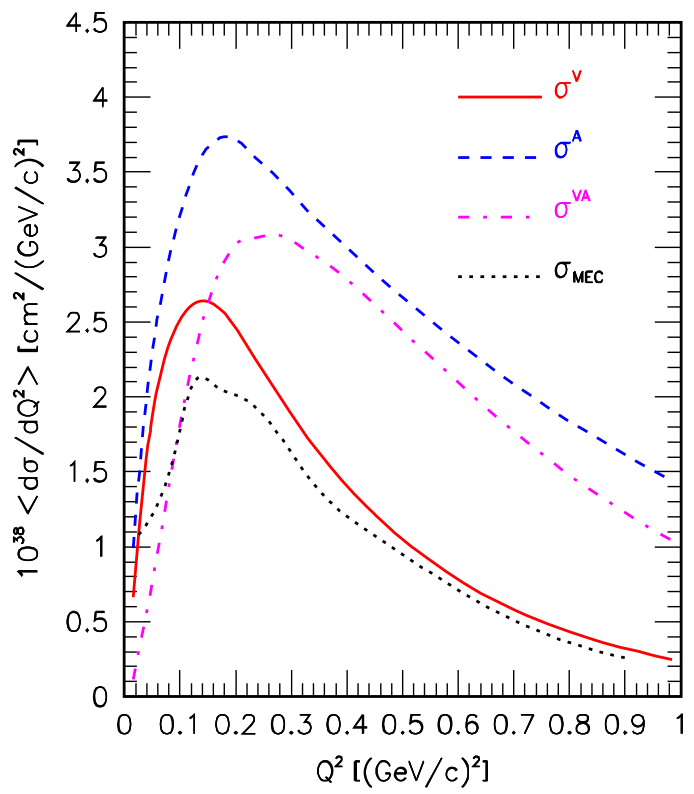

FIG. 1. Flux-integrated $\left\langle\sigma^{V}\right\rangle$ (solid line), $\left\langle\sigma^{A}\right\rangle$ (dashed line), $\left\langle\sigma^{V A}\right\rangle$ (dashed-dotted line), and $\left\langle d \sigma_{\mathrm{MEC}} / d Q^{2}\right\rangle$ (dotted line) cross sections of $\nu_{\mu}$ scattering on ${ }^{12} \mathrm{C}$ as functions of $Q^{2}$.

antineutrino sample, the purity of the antineutrino CCQElike sample is $61 \%$.

To extract the values of the axial form factor $F_{A}\left(Q^{2}\right)$ as a function of $Q^{2}$, using the measured neutrino flux-integrated $\left\langle d \sigma / d Q^{2}\right\rangle$ cross section, we calculated the $\left\langle\sigma^{V}\right\rangle,\left\langle\sigma^{A}\right\rangle$, $\left\langle\sigma^{V A}\right\rangle$, and $\left\langle d \sigma_{\mathrm{MEC}} / d Q^{2}\right\rangle$ cross sections with the booster neutrino beam line $\nu_{\mu}$ flux [9]. In Fig. 1, these cross sections for $\nu_{\mu}$ scattering on ${ }^{12} \mathrm{C}$ are shown against $Q^{2}$. In Fig. 2(a), we show the measured flux-integrated $\left\langle d \sigma / d Q^{2}\right\rangle$ as a function of $Q^{2}$. To extract the values of $F_{A}$, this cross section with the shape-only error was used in Eq. (16). Reference [62] shows that the best-fit parameter values are not significantly altered by including the MiniBooNE normalization uncertainties in the CCQE fit. The results, $F_{A}\left(Q^{2}\right)$ as a function of $Q^{2}$, are shown in Fig. 2(b). Also shown in this figure are the results from Ref. [49], obtained within the RDWIA, i.e., without the $2 p-2 h$ MEC contributions. The axial form factor values extracted in the RDWIA approach agree well with the dipole parametrization with $M_{A}=1.36 \mathrm{GeV}$. As observed, results in Fig. 2(b) clearly show the relevant role played by the $2 p-2 h$ MEC contributions. The values of $F_{A}$ obtained with the RDWIA are higher and decrease with $Q^{2}$ more slowly than corresponding values extracted within the RDWIA + MEC approach. The fits were made to the singledifferential $d \sigma / d Q^{2}$ (1D fit) and double-differential $d^{2} \sigma / d T d \cos \theta$ (2D fit) cross sections, using the $\chi^{2}$ statistic

$\chi_{1 D}^{2}=\sum_{k=1}^{N}\left[\frac{\left(d \sigma / d Q_{\mathrm{QE}}^{2}\right)_{k}^{\text {data }}-\left(d \sigma / d Q^{2}\right)_{k}^{\text {th }}}{\Delta\left(d \sigma / d Q^{2}\right)_{k}}\right]^{2} \rightarrow 1 \mathrm{D}$
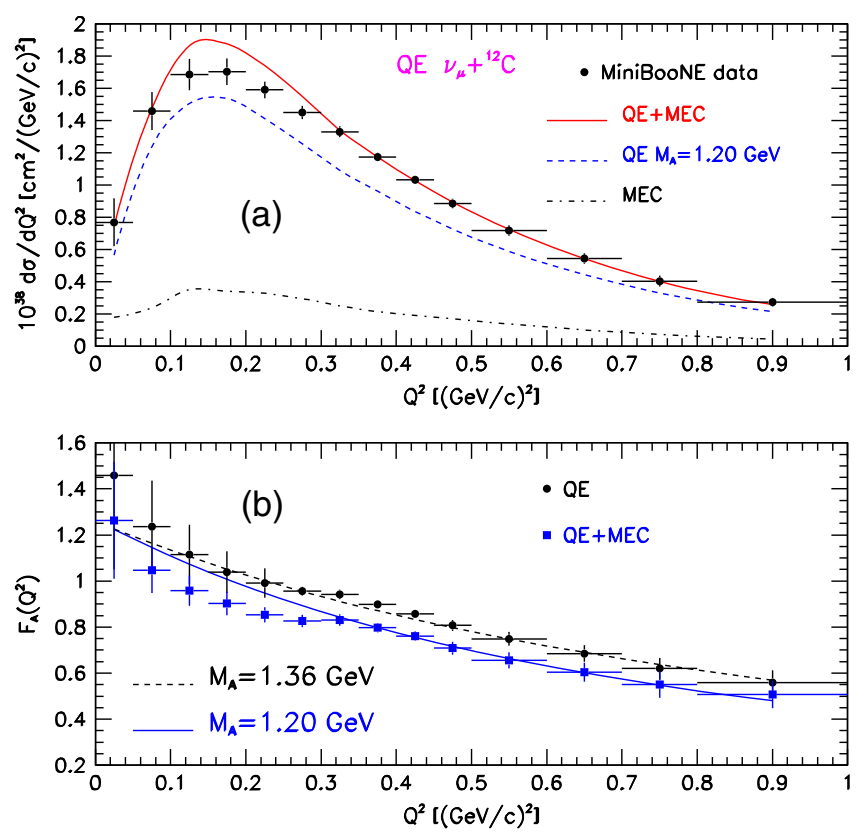

FIG. 2. Flux-integrated $d \sigma / d Q^{2}$ cross section per target neutron for the $\nu_{\mu}$ CCQE-like scattering (upper panel) and axial form factor $F_{A}\left(Q^{2}\right)$ extracted from the MiniBooNE data as functions of $Q^{2}$. Upper panel: Calculations from the RDWIA + MEC (solid line), RDWIA (dashed line) with $M_{A}=1.20 \mathrm{GeV}$, and $2 p-2 h$ MEC contributions (dashed-dotted line). Lower panel: Filled squares (filled circles) are the axial form factor extracted within the RDWIA + MEC (RDWIA), and the solid (dashed) line is the result of the dipole parametrization with $M_{A}=$ $1.20(1.36) \mathrm{GeV}$.

$$
\begin{aligned}
\chi_{2 D}^{2} & =\sum_{l=1}^{M}\left[\frac{\left(d^{2} \sigma / d T d \cos \theta\right)_{l}^{\mathrm{data}}-\left(d^{2} \sigma / d T d \cos \theta\right)_{l}^{\mathrm{th}}}{\Delta\left(d^{2} \sigma / d T d \cos \theta\right)_{l}}\right]^{2} \\
& \rightarrow 2 \mathrm{D},
\end{aligned}
$$

where $\left(d \sigma / d Q^{2}\right)^{\text {th }}$ and $\left(d \sigma / d Q_{\mathrm{QE}}^{2}\right)^{\text {data }}$ are functions of $Q^{2}$ and $Q_{\mathrm{QE}}^{2}$, respectively, and $\Delta\left(d \sigma / d Q^{2}\right)_{k}$ and $\Delta\left(d^{2} \sigma / d T d \cos \theta\right)_{l}$ are the diagonals of the MiniBooNE shape-only covariance matrices for neutrino results. The following best-fit $\chi^{2}$ and $M_{A}$ values are obtained: $\chi_{1 D}^{2} / \mathrm{DOF}=19 / 13$ and $M_{A}=1.17 \pm 0.03 \mathrm{GeV}$ for the $1 \mathrm{D}$ fit and $\chi_{2 D}^{2} / \mathrm{DOF}=62 / 136$ and $M_{A}=1.24 \pm$ $0.09 \mathrm{GeV}$ for the 2D fit. The MiniBooNE $d \sigma / d Q_{\mathrm{QE}}^{2}$ and $d \sigma / d T d \cos \theta$ cross sections are statistically not independent, and the result of the 2D fit is preferred as it contains the most information and has minimal model dependence. However, the best-fit value of $M_{A}=1.20 \pm 0.06 \mathrm{GeV}$ $\left(\chi^{2} / \mathrm{DOF}=111 / 150\right)$ for the $1 \mathrm{D}+2 \mathrm{D}$ fit also is obtained for completeness. Although there is a difference between the best-fit $M_{A}$ values, the errors from the fits cover this difference. We round off the values 1.17 and 1.24 to the value of $M_{A}=1.20 \mathrm{GeV}$ and use it in our calculation. This $M_{A}$ value is in agreement within the errors with the best-fit 

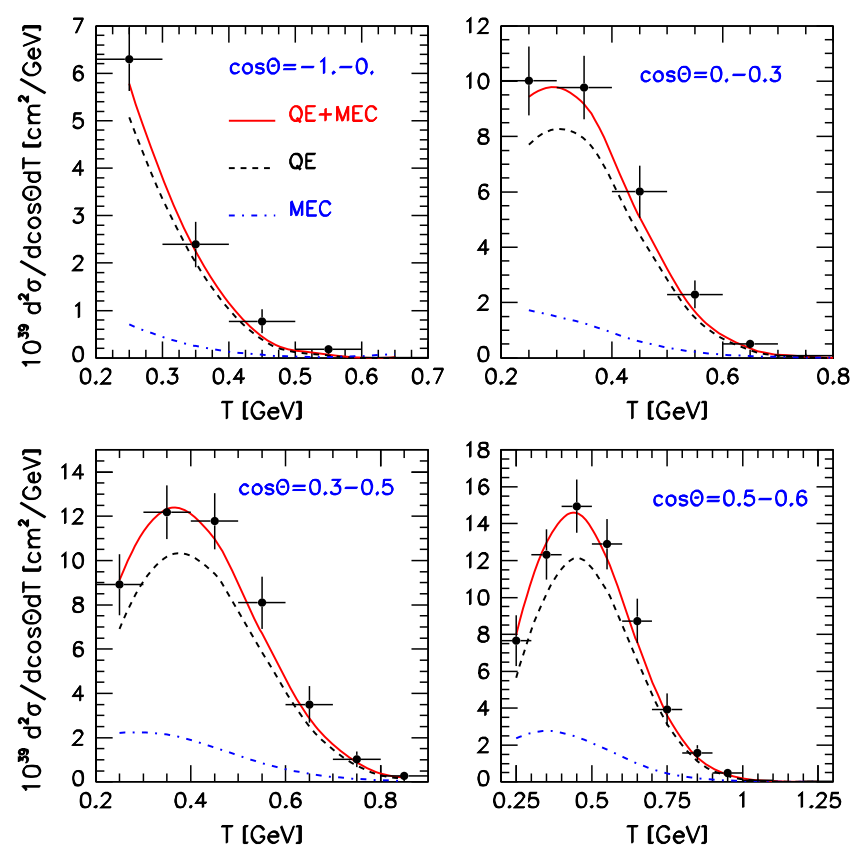

FIG. 3. Flux-integrated $d^{2} \sigma / d T d \cos \theta$ cross section per target neutron for the $\nu_{\mu}$ CCQE-like scattering as a function of muon kinetic energy for the four muon scattering angle bins: $\cos \theta=(-1-0),(0-0.3),(0.3-0.5)$, and (0.5-0.6). As shown in the key, cross sections were calculated within the RDWIA + MEC approach. The QE and $2 p-2 h$ MEC contributions are also presented separately. The MiniBooNE data are shown as points with the shape error only.

value of $M_{A}=1.15 \pm 0.03 \mathrm{GeV}$ obtained from the main CCQE fit of the MiniBooNE and MINERvA data in Refs. [48,62]. Additionally, Ref. [62] obtained the bestfit value of $M_{A}=1.15 \pm 0.09 \mathrm{GeV}$ for the $1 \mathrm{D}$ fit to the MiniBooNE neutrino data (Table C10) with $\left(d \sigma / d Q_{\mathrm{QE}}^{2}\right)^{\text {data }}$ and $\left(d \sigma / d Q^{2}\right)^{\text {th }}$ cross sections as functions of $Q_{\mathrm{QE}}^{2}$. This $M_{A}$ value within the errors is in agreement with our 1D fit result.

The best-fit $d \sigma / d Q^{2}$ distribution is compared with the data in Fig. 2(a). The result of the dipole parametrization of $F_{A}\left(Q^{2}\right)$ with $M_{A}=1.20 \mathrm{GeV}$ is shown in Fig. 2(b). There is an overall agreement between the RDWIA+ MEC $d \sigma / d Q^{2}$ cross section and the data, but the model slightly overestimates the data in the range $0.08<Q^{2}<$ $0.3(\mathrm{GeV} / c)^{2}$. A downside of using the CCQE-corrected data is explicit subtraction of $\pi$-less $\Delta$ decay events (simulated with a neutrino generator) in the MiniBooNE analysis. On the other hand, in our calculation, this channel gives a contribution to the MEC cross sections because there is no obvious way to account for this effect. We note that Refs. [37,48,62] also used the CCQE-corrected data to evaluate the value of $M_{A}$. In Figs. 3-7, we show double-differential cross sections calculated with $M_{A}=1.20 \mathrm{GeV}$. The results for $d^{2} \sigma /$ $d T d \cos \theta$ cross sections against the kinematic energy of
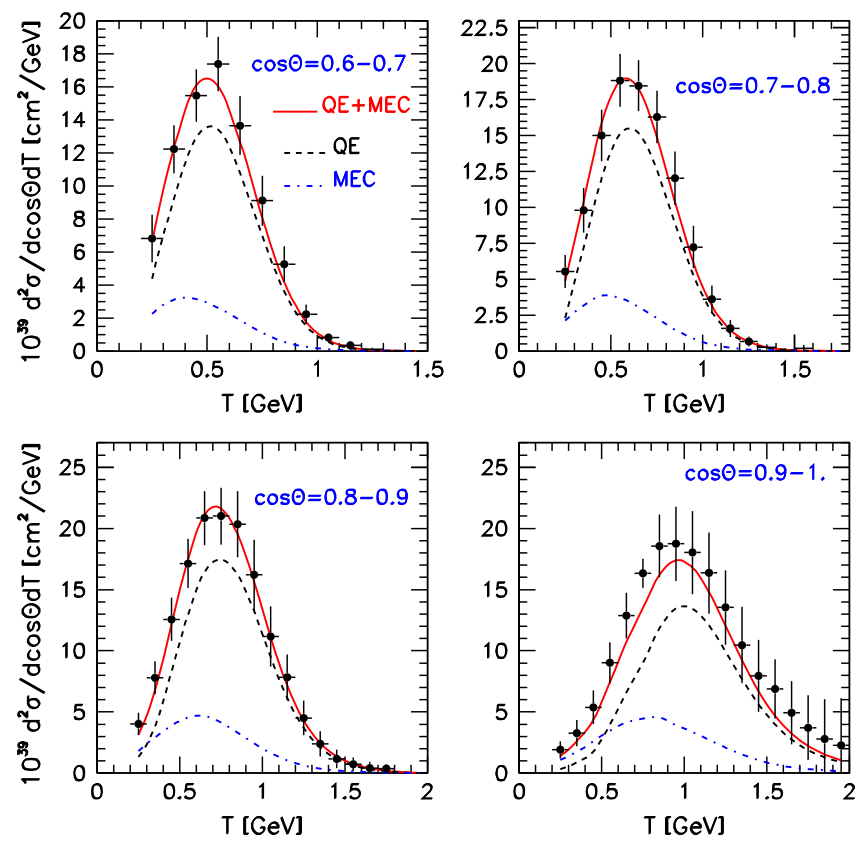

FIG. 4. Same as Fig. 3, but for muon scattering angle bins: $\cos \theta=(0.6-0.7),(0.7-0.8),(0.8-0.9)$, and (0.9-1).

the muon are shown in Figs. 3 and 4. We present a large variety of kinematical situations in which each panel refers to results averaged over a particular muon angular bin. As observed, the model tends to slightly underestimate the data for most forward angles, i.e., $0.9<\cos \theta<1$. As the scattering angle increases, the RDWIA + MEC prediction agrees well with the data. Results in Figs. 3 and 4 clearly show that the $2 p-2 h$ MEC contributions are essential in order to describe data. The contributions of these effects are comparable with the genuine QE process, being of order $25 \%$ and increasing up to $30 \%$ at low $Q^{2}$. In Figs. 5-7, we present the results averaged over the muon kinetic energy bins as functions of the muon scattering angle. These graphs complement the previous ones and show that the RDWIA + MEC model is able to reproduce the data. There is good agreement between the calculated results and the data within experimental error. In the region $0.2<T<0.3 \mathrm{GeV}$ and $-1<\cos \theta<-0.2$, the model result is slightly lower than the measured cross section, and the difference decreases with muon energy. In Fig. 8, results are presented for the MiniBooNE fluxintegrated CCQE-like $d \sigma / d T$ differential cross section as a function of the muon kinetic energy (upper panel) and $d \sigma / d \cos \theta$ cross section vs of muon scattering angle (lower panel). The measured $d \sigma / d T(d \sigma / d \cos \theta)$ cross section with the shape-only error was obtained by summing the double-differential cross section over $\cos \theta$ bins ( $T$ bins) presented in Tables VI and VII in Ref. [9]. The integration over muon kinetic energy has been performed in the range $0.2<T<2 \mathrm{GeV}$. As shown, 

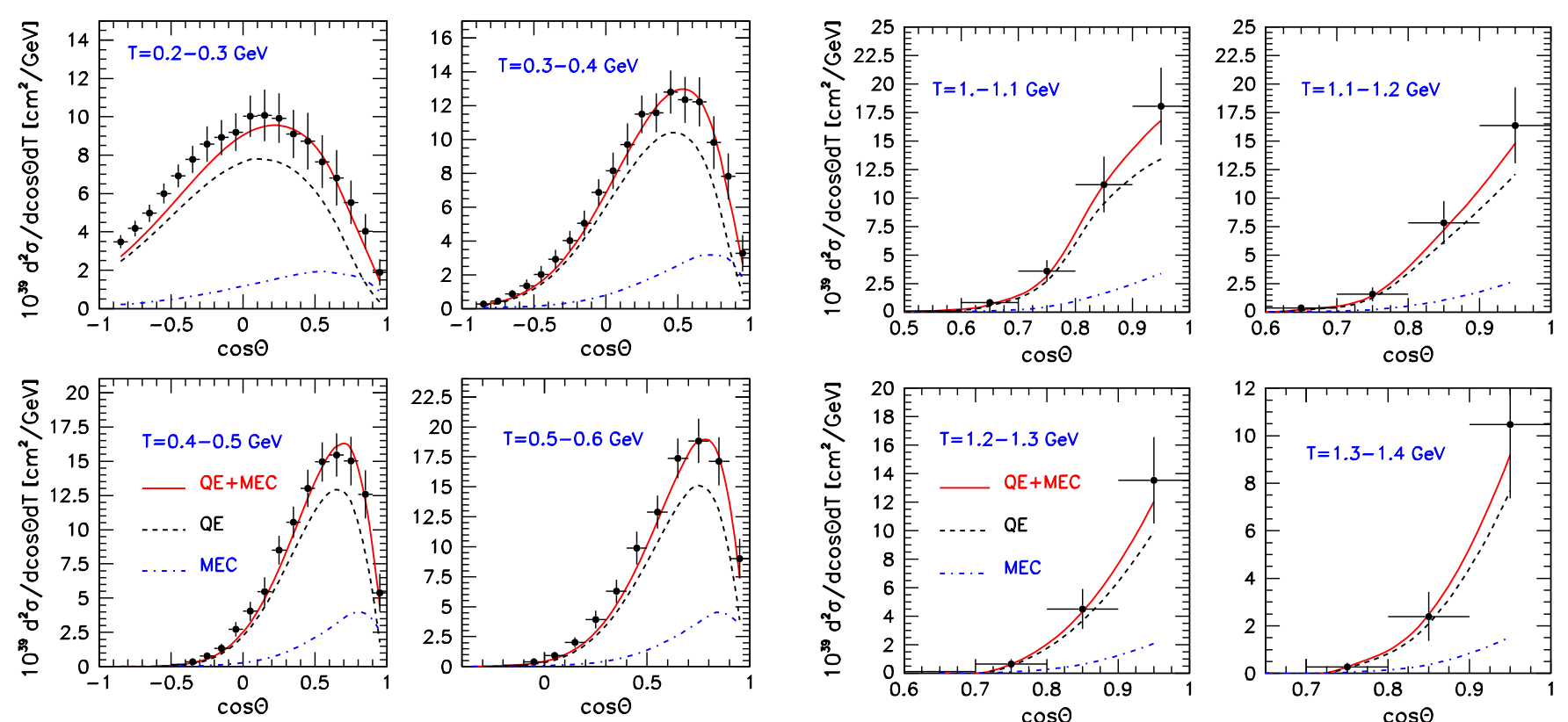

FIG. 5. Flux-integrated $d^{2} \sigma / d T d \cos \theta$ cross section per target neutron for the $\nu_{\mu}$ CCQE-like scattering as a function of $\cos \theta$ for the four muon kinetic energy bins: $T(\mathrm{GeV})=(0.2-0.3)$, $(0.3-0.4),(0.4-0.5)$, and (0.5-0.6). As shown in the key, cross sections were calculated within the RDWIA + MEC approach. The QE and $2 p-2 h$ MEC contributions are also presented. The MiniBooNE data are shown as points with the shape error only.

the RDWIA + MEC model with $M=1.20 \mathrm{GeV}$ is capable of reproducing the magnitude as well as the shape of the experimental cross sections. The difference between the calculated and measured $d \sigma / d Q^{2}, d \sigma / d T$,
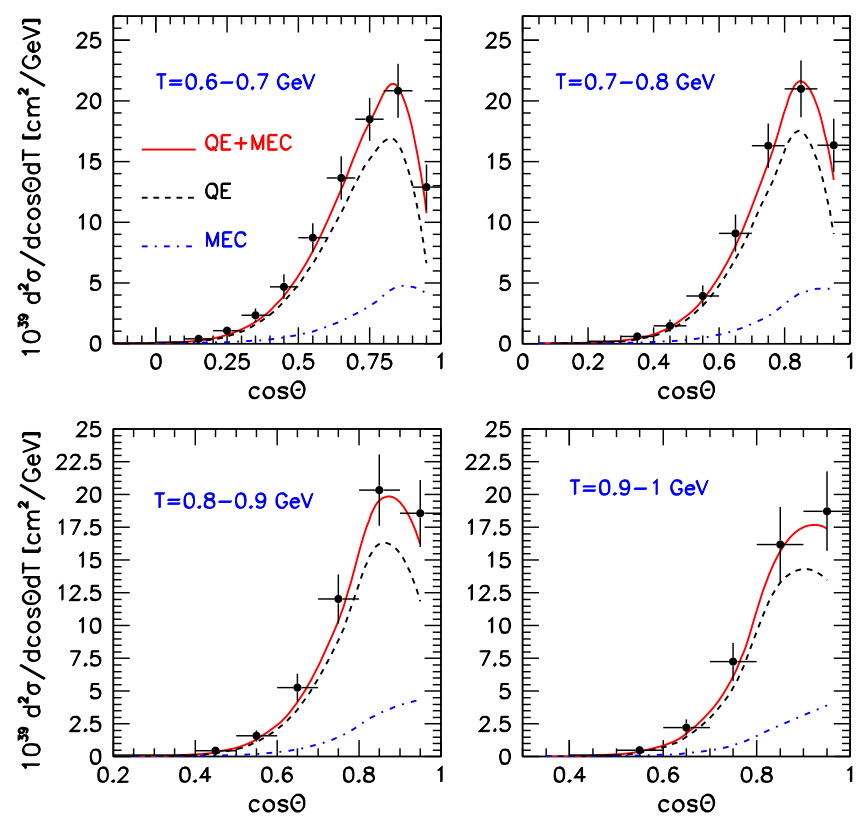

FIG. 6. Same as Fig. 5, but for the four muon kinetic energy bins: $T(\mathrm{GeV})=(0.6-0.7),(0.7-0.8),(0.8-0.9)$, and $(0.9-1)$.

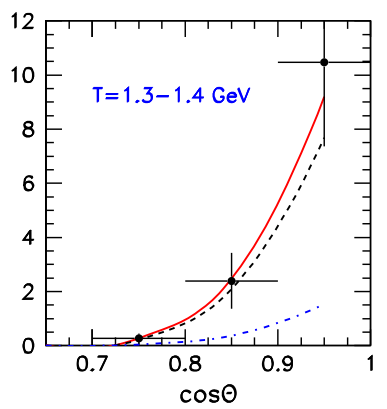

FIG. 7. Same as Fig. 5, but for the four muon kinetic energy bins: $T(\mathrm{GeV})=(1 .-1.1),(1.1-1.2),(1.2-1.3)$, and (1.3-1.4).
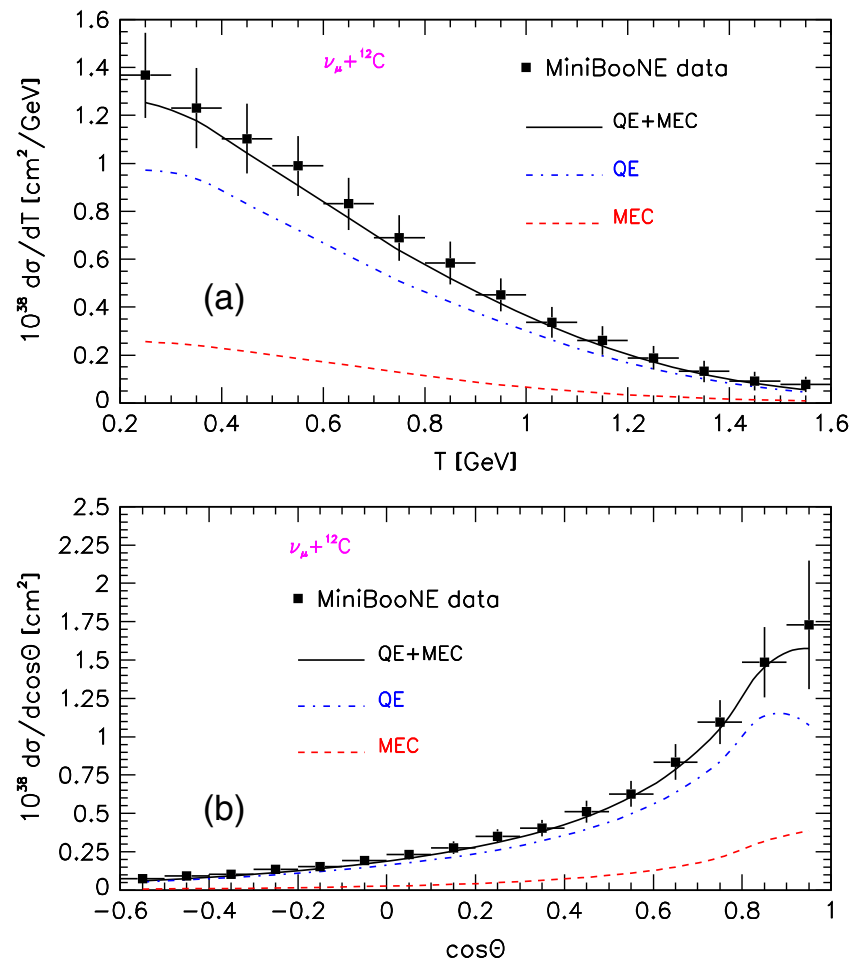

FIG. 8. Flux-integrated $d \sigma / d T$ cross section as a function of muon kinetic energy (upper panel) and $d \sigma / d \cos \theta$ cross section for $T>0.2 \mathrm{GeV}$ as a function of muon scattering angle (lower panel) for the $\nu_{\mu}$ CCQE-like scattering per target neutron. As shown in the key, cross sections were calculated within the RDWIA + MEC. The RDWIA and $2 p-2 h$ MEC results are also presented separately. The MiniBooNE data are shown as points with the shape error only. 


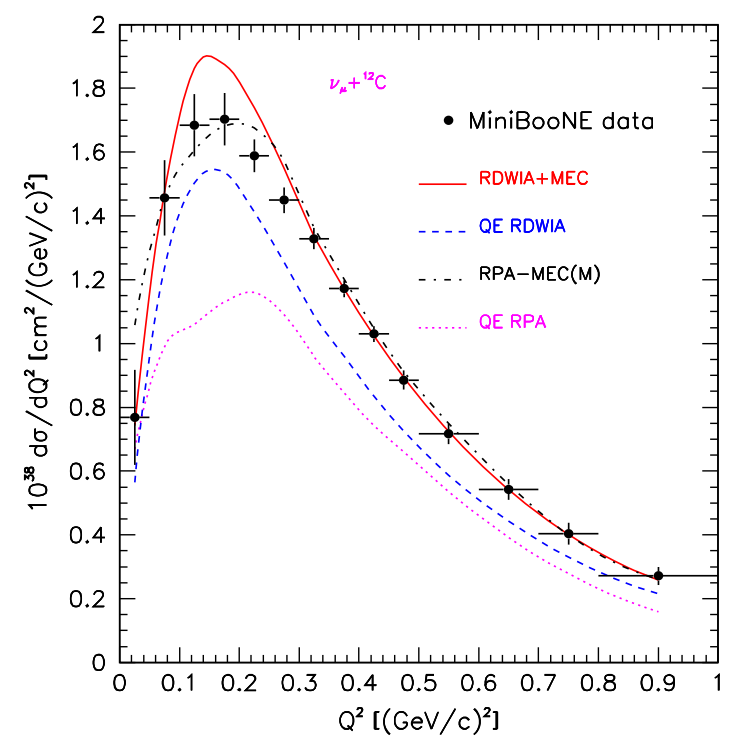

FIG. 9. Flux-integrated $d \sigma / d Q^{2}$ cross section per target neutron for the $\nu_{\mu}$ CCQE-like and CCQE processes as a function of $Q^{2}$. As shown in the key, cross sections were calculated within the RDWIA + MEC $\left(M_{A}=1.20 \mathrm{GeV}\right)$ and RPA-MEC [35] models. The CCQE contributions calculated in the RDWIA and RPA approaches are also presented separately. The MiniBooNE data are shown as points with the shape-only error.

and $d \sigma / d \cos \theta$ flux-integrated cross sections is less than $\pm 12 \%$. Therefore, a cautious estimate of the uncertainty of the CCQE-like one-differential cross sections calculated within the RDWIA + MEC approach is of the order of $\pm 12 \%$.

\section{B. Comparison of the results of the RDWIA + MEC, RPA-MEC, and SuSAv2-MEC models}

In Figs. 9-11, the MiniBooNE neutrino flux-averaged CCQE-like differential cross sections calculated within the different approached are presented. In Fig. 9, we show the $d \sigma / d Q^{2}$ cross sections as measured in Ref. [9] and as calculated in the RDWIA + MEC and RPA-MEC [35] models. Also shown are CCQE cross sections obtained in these approaches. From the figure, one can observe that these calculations describe well the experimental data at $Q^{2}>0.3(\mathrm{GeV} / c)^{2}$. The RDWIA + MEC model slightly overestimates the data in the range $0.08<Q^{2}<$ $0.3(\mathrm{GeV} / c)^{2}$, and in the case of the RPA + MEC approach, a tendency to overestimate the data appears at low $Q^{2}<0.06(\mathrm{GeV} / c)^{2}$. In the range of $Q^{2}<$ $0.3(\mathrm{GeV} / c)^{2}$, which is affected by RPA quenching, the CCQE cross sections calculated in the RDWIA with $M_{A}=1.20 \mathrm{GeV}$ is about $30 \%$ larger than those obtained in Ref. [35], and the discrepancy decreases with $Q^{2}$ up to $12 \%$ at $Q^{2} \approx 0.9(\mathrm{GeV} / c)^{2}$. In Fig. 10, we show the double-differential $d^{2} \sigma / d T d \cos \theta$ cross sections calculated in the RDWIA + MEC, SuSAv2-MEC [61], and
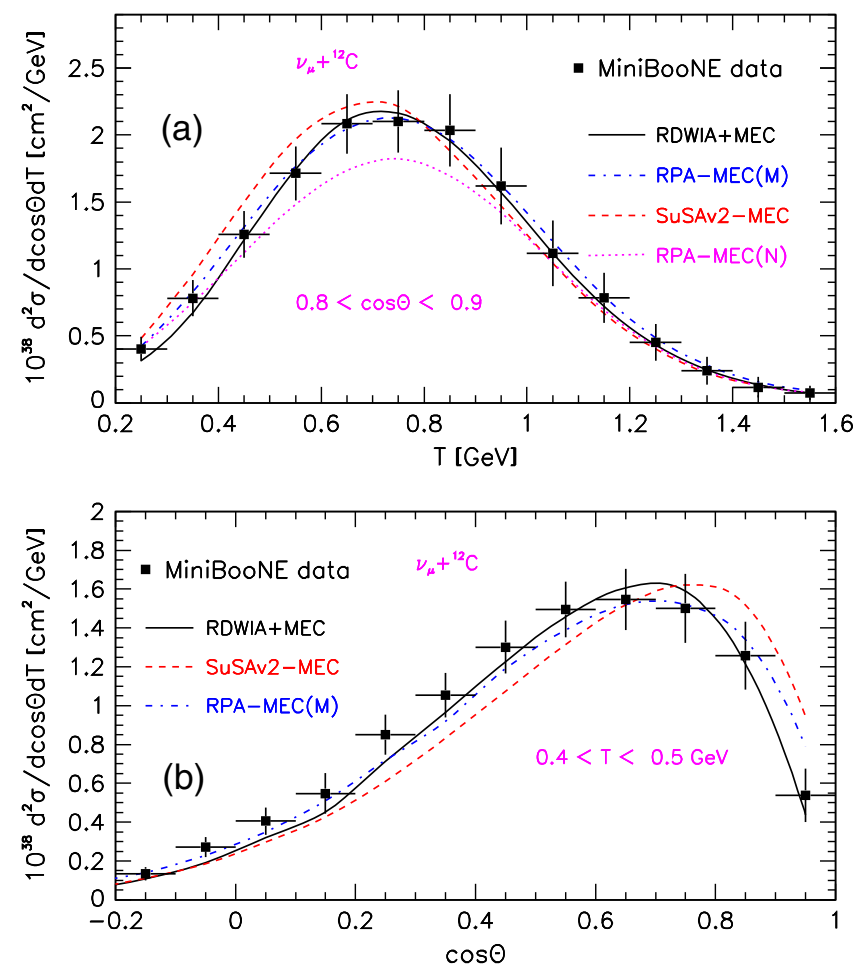

FIG. 10. Flux-integrated $d^{2} \sigma / d T d \cos \theta$ cross section per target neutron for the CCQE-like scattering. Upper panel: Cross sections calculated within the RDWIA + MEC (solid line), RPA-MEC (dashed-dotted line) [35], SuSAv2-MEC (dashed line), and RPA-MEC (dotted line) [37] models for $0.8<\cos \theta<$ 0.9 as functions of muon kinetic energy. Lower panel: Cross sections calculated in the RDWIA + MEC (solid line), SuSAv2MEC (dashed line), and RPA-MEC (dashed-dotted line) [35] approaches for $0.4<T<0.5 \mathrm{GeV}$ as functions of muon scattering angle. The MiniBooNE data are shown as points with the shape-only error.

RPA-MEC [35,37] approaches. For the sake of illustration in Fig. 10(a), the results are given for $0.8<\cos \theta<$ 0.9 as functions of the muon kinetic energy. As one can observe, the results of the RDWIA + MEC, SuSAv2MEC, and RPA-MEC [35] models are in agreement with data. In the case of the RPA-MEC result [37], a tendency to underestimate the MiniBooNE data appears. In Fig. 10(b), the results are given for muon kinetic energy bin $0.4<T<0.5 \mathrm{GeV}$ as functions of the muon scattering angle. As shown, the results obtained within the RDWIA + MEC and RPA-MEC [35] models agree well with data. On the other hand, a difference between the SuSAv2-MEC result and data is observed. The fluxaveraged differential cross sections $d \sigma / d T$ and $d \sigma / d \cos \theta$ (for $T>0.2 \mathrm{GeV}$ ), calculated in the RDWIA + MEC, SuSAv2-MEC, and RPA-MEC [35] approaches, are presented in Fig. 11, which shows $d \sigma / d T$ as a function of muon kinetic energy and $d \sigma / d \cos \theta$ as a function of the muon scattering angle. Also shown are the MiniBooNE measured cross sections with the shape-only 

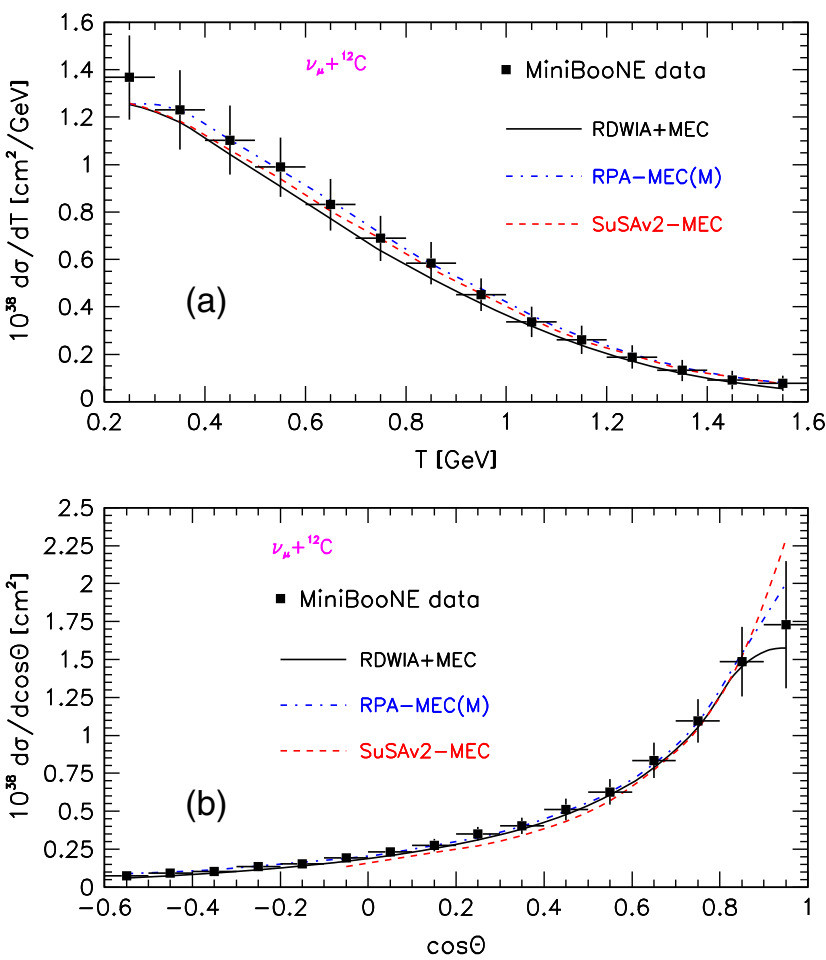

FIG. 11. Flux-integrated $d \sigma / d T$ cross section as a function of muon kinetic energy (upper panel) and $d \sigma / d \cos \theta$ cross section for $T>0.2 \mathrm{GeV}$ as a function of muon scattering angle (lower panel) for the CCQE-like scattering per target neutron. As shown in the key, cross sections were calculated within the RDWIA+ MEC, SuSAv2-MEC, and RPA-MEC [35] models. The MiniBooNE data are shown as points with the shape-only error.

error. There is a good agreement within the errors between the calculated results and data.

\section{Calculation of neutrino CCQE-like differential cross sections at energies of the NOvA experiment}

Within the RDWIA + MEC approach with $M_{A}=$ 1.20 GeV, which describes the MiniBooNE neutrino data well, we estimated the neutrino CCQE-like flux-integrated differential cross sections at energies available at the NOvA experiment [51,52]. The NOvA detectors are situated $14 \mathrm{mrad}$ off the neutrino main injector beam axis, so they expose a relatively narrow band approximately $0.5-5 \mathrm{GeV}$ of neutrino energies, centered at $2 \mathrm{GeV}$ [63]. This flux is used in the calculation of the NOvA flux-integrated differential cross sections.

In the fiducial region of the NOvA near detector (ND), the liquid scintillator $\left(\mathrm{CH}_{2}\right)$ comprises $63 \%$ of the detector mass. The mass weight for this detector component is as follows: ${ }^{12} \mathrm{C}$ is $66.8 \%,{ }^{35} \mathrm{Cl}$ is $16.4 \%,{ }^{1} \mathrm{H}$ is $10.5 \%,{ }^{48} \mathrm{Ti}$ is $3.3 \%,{ }^{16} \mathrm{O}$ is $2.6 \%$, and others are $0.4 \%$ [64]. So, the ND is dominated by carbon, chlorine, and hydrogen. We assumed that the NOVA CCQE-like scattering sample consists of two processes: scattering on ${ }^{12} \mathrm{C}$ and ${ }^{35} \mathrm{Cl}$. The mass weight of carbon $\alpha_{c}$ and chlorine $\alpha_{C l}$ was rescaled for neutrino scattering as $\alpha_{C}=0.806, \alpha_{C l}=0.194$, and $\alpha_{c}+\alpha_{C l}=1$. In Ref. [65], we calculated within the RDWIA the CCQE differential cross sections for (anti)neutrino scattering on ${ }^{40} \mathrm{Ar}$. The difference between the nuclear structures of ${ }^{40} \mathrm{Ar}$ and ${ }^{35} \mathrm{Cl}$ is not significant. Therefore, the NOvA CCQE-like differential cross sections per target neutron were estimated for neutrino scattering on carbon and argon; herewith, the $2 p-2 h$ MEC contributions for ${ }^{40} \mathrm{Ar}$ were calculated using the parametrizations for ${ }^{12} \mathrm{C}$ rescaled for argon according to Ref. [66]. Then, the NOvA neutrino scattering cross section per target neutron, i.e., the cross sections averaged over the ND mass weight, can be expressed as $\sigma_{M I X}=\alpha_{C} \sigma_{C}+\alpha_{C l} \sigma_{A r}$, where $\sigma_{c}\left(\sigma_{A r}\right)$ is the cross section per target neutron of neutrino scattering on ${ }^{12} \mathrm{C}\left({ }^{40} \mathrm{Ar}\right)$. The uncertainty in the $\sigma_{M I X}$ due to approximation of the cross section per neutron for chlorine by the cross section for argon and neglect of contributions of the other nuclei is about of $0.6 \%$. Figure 12 contains the RDWIA + MEC model predictions corresponding to the NOvA flux-integrated $d \sigma / d Q^{2}$ cross sections per target neutron for neutrino scattering on ${ }^{12} \mathrm{C}$ and ${ }^{40} \mathrm{Ar}$. The cross sections were calculated for the CCQE and $2 p-2 h$ MEC
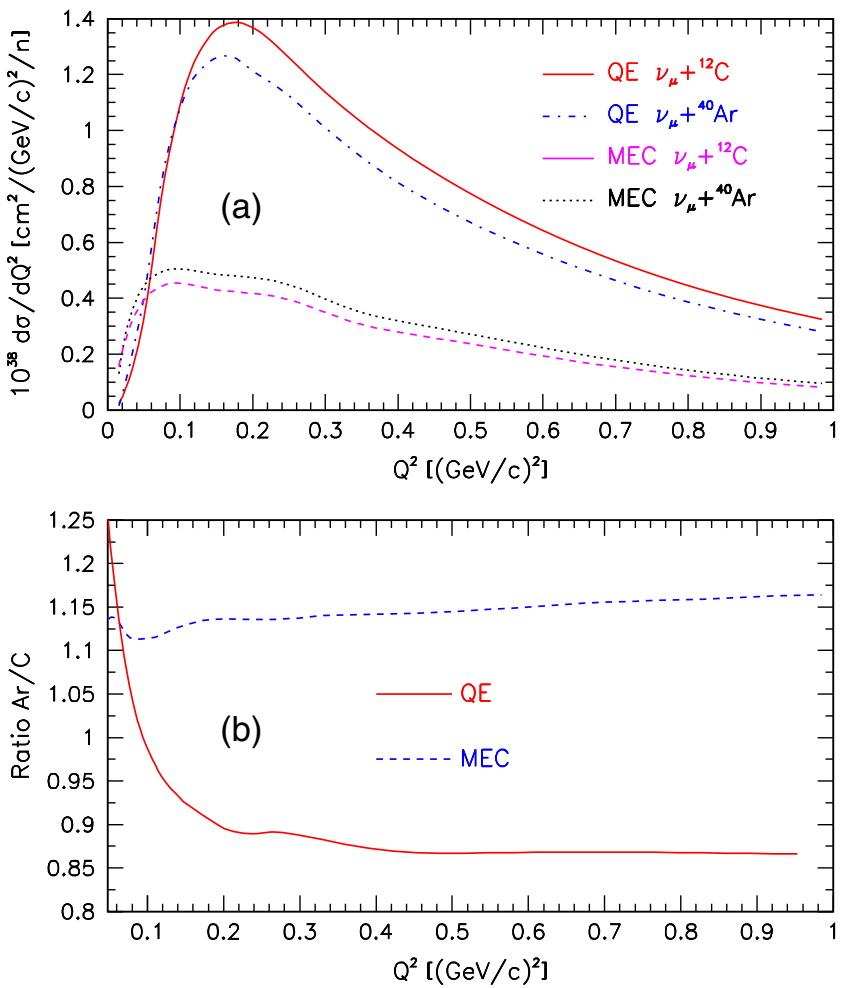

FIG. 12. NOvA flux-integrated $d \sigma / d Q^{2}$ cross sections per target neutron for the $\nu_{\mu} \mathrm{CCQE}$ and $2 p-2 h$ MEC scattering (upper panel) and ratio $R_{\mathrm{QE}}$ and $R_{\mathrm{MEC}}$ (lower panel) as functions of $Q^{2}$. As shown in the key, cross sections were calculated for neutrino scattering on ${ }^{12} \mathrm{C}$ and ${ }^{40} \mathrm{Ar}$. 


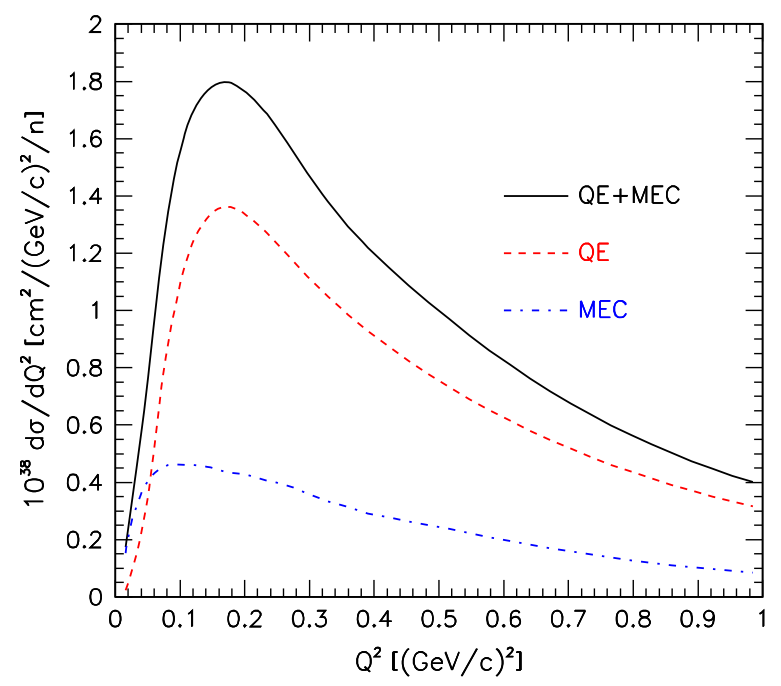

FIG. 13. NOvA flux-integrated $d \sigma / d Q^{2}$ cross sections per target neutron for the $\nu_{\mu}$ CCQE-like scattering (solid line). The CCQE (dashed line) and $2 p-2 h$ MEC (dashed-dotted line) results are also presented separately. The cross sections are shown as functions of $Q^{2}$.
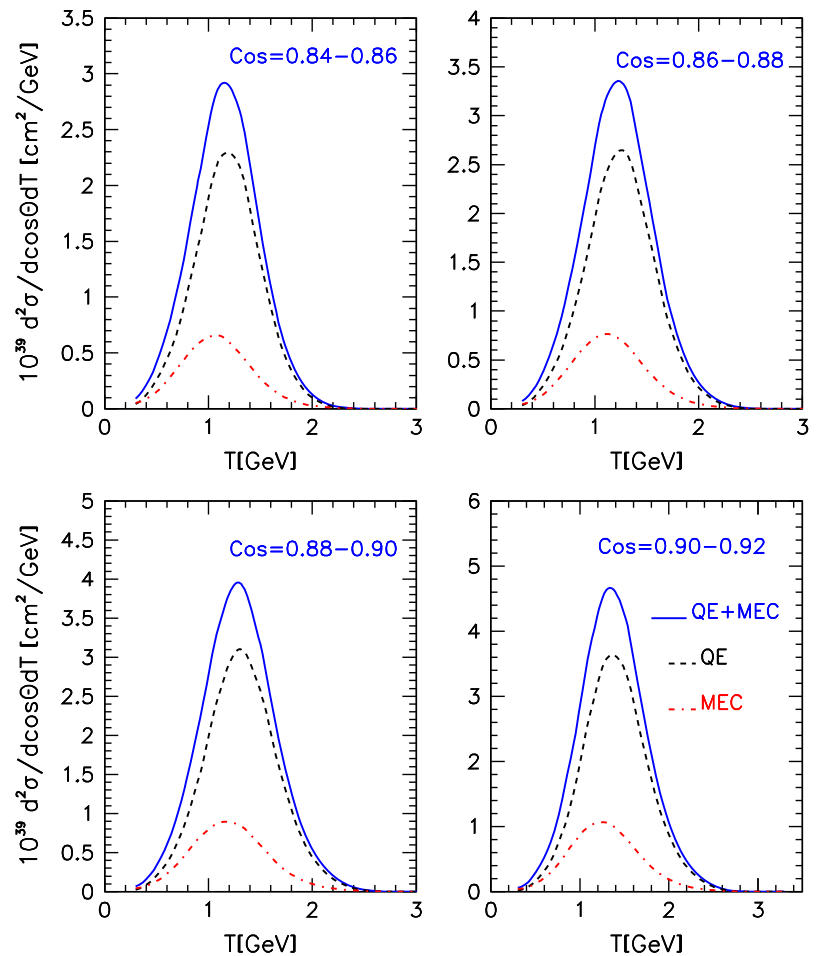

FIG. 14. NOvA flux-integrated $d^{2} \sigma / d \cos \theta d T$ cross section per target neutron for the $\nu_{\mu}$ CCQE-like scattering as a function of muon kinetic energy for the four muon scattering angle bins: $\cos \theta=(0.84-0.86),(0.86-0.88),(0.88-0.90)$, and (0.90-0.92). As shown in the key, the cross section was calculated within the RDWIA + MEC. The CCQE and $2 p-2 h$ MEC contributions are also shown separately. processes in the region $T>0.3 \mathrm{GeV}$ and $0.3<\cos \theta<1$. Also shown are the ratios $R_{\mathrm{QE}}=\left(d \sigma / d Q^{2}\right)_{\mathrm{QE}}^{A r} /\left(d \sigma / d Q^{2}\right)_{\mathrm{QE}}^{C}$ and $R_{\mathrm{MEC}}=\left(d \sigma / d Q^{2}\right)_{\mathrm{MEC}}^{A r} /\left(d \sigma / d Q^{2}\right)_{\mathrm{MEC}}^{C}$, where $(d \sigma /$ $\left.d Q^{2}\right)_{\mathrm{QE}}^{A r}\left[\left(d \sigma / d Q^{2}\right)_{\mathrm{QE}}^{C}\right]$ and $\left(d \sigma / d Q^{2}\right)_{\mathrm{MEC}}^{A r}\left[\left(d \sigma / d Q^{2}\right)_{\mathrm{MEC}}^{C}\right]$ are the CCQE and $2 p-2 h$ MEC cross sections per target neutron for neutrino scattering on ${ }^{40} \mathrm{Ar}\left({ }^{12} \mathrm{C}\right)$, respectively. The figure clearly shows that the ratio $R_{\mathrm{QE}}$ reduces with $Q^{2}$ from 1.2 at $Q^{2} \approx 0.04(\mathrm{GeV} / c)^{2}$ up to 0.87 at $Q^{2} \approx 1(\mathrm{GeV} / c)^{2}$. On the other hand, the ratio $R_{\mathrm{MEC}}$ increases slowly with $Q^{2}$ from 1.1 at $Q^{2} \approx 0.1(\mathrm{GeV} / c)^{2}$ up to 1.17 at $Q^{2} \approx 1(\mathrm{GeV} / c)^{2}$. Thus, the RDWIA + MEC model predicts that the CCQE differential cross section per target neutron reduces and the $2 p-2 h$ MEC contribution increases with the mass number of the target.

The results in Fig. 13 correspond to the flux-integrated NOvA $\left(d \sigma / d Q^{2}\right)_{M I X}$ cross section per target neutron of the CCQE-like neutrino scattering. Also shown are the contributions of the CCQE and $2 p-2 h$ MEC processes. The ratio $R=\left(d \sigma / d Q^{2}\right)_{M I X} /\left(d \sigma / d Q^{2}\right)_{C}$ is about 0.98 in the range $0.1<Q^{2}<1(\mathrm{GeV} / c)^{2}$; i.e., the NOvA CCQElike cross section per target nucleon for neutrino scattering in the NOvA ND is, practically, the same as the one for scattering on carbon.
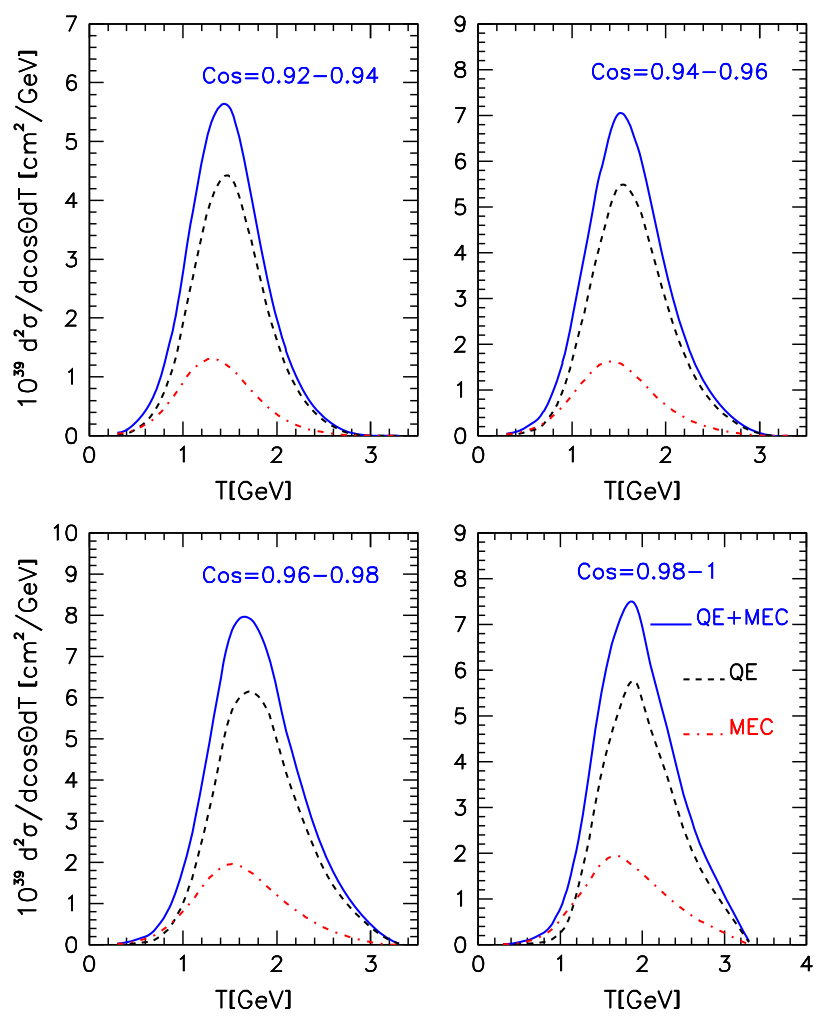

FIG. 15. Same as Fig. 14, but for muon scattering angle bins: $\cos \theta=(0.92-0.94),(0.94-0.96),(0.96-0.98)$, and (0.98-1). 

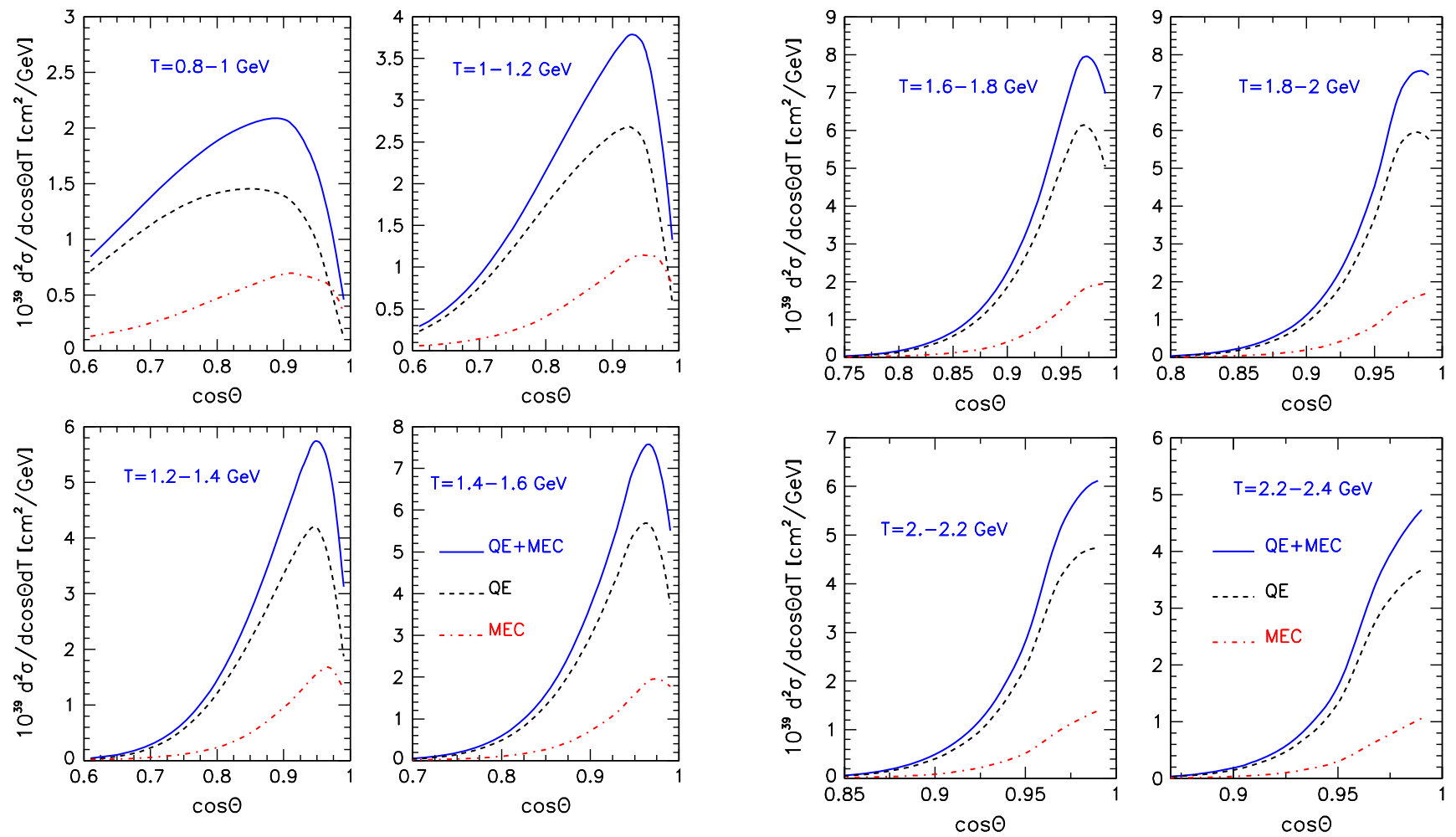

FIG. 16. NOvA flux-integrated $d^{2} \sigma / d \cos \theta d T$ cross section per target neutron for the $\nu_{\mu}$ CCQE-like scattering as a function of muon kinetic energy for the four muon kinetic energy bins: $T(\mathrm{GeV})=(0.8-1),(1-1.2),(1.2-1.4)$, and (1.4-1.6). As shown in the key, the cross section was calculated within the RDWIA + MEC. The CCQE and $2 p-2 h$ MEC contributions are also presented separately.

In Figs. 14 and 15, we present the flux-integrated CCQE-like double-differential cross sections per neutron predicted for the NOvA experiment. The graphs are plotted against the muon kinetic energy, and each panel corresponds to a bin in the muon scattering angle. The double-differential cross sections averaged over muon kinetic energy bins are shown in Figs. 16 and 17. In these figures, we show the separate contributions of the genuine $\mathrm{QE}$ and $2 p-2 h$ MEC processes. The NOvA fluxintegrated CCQE-like $\nu_{\mu}$ cross sections per target neutron $d \sigma / d T$ as a function of the muon kinetic energy and $d \sigma / d \cos \theta$ vs muon scattering angle are presented in Fig. 18. The pure QE and $2 p-2 h$ MEC results are also shown separately. Integration of the double-differential cross section over the muon scattering angle has been performed in the range $0.6<\cos \theta<1$ for calculation of the $d \sigma / d T$ cross section and over muon kinetic energy in the range $0.2<T<3.5 \mathrm{GeV}$ for evaluation of the $d \sigma / d \cos \theta$ cross section. We suggest that the uncertainties in these cross sections are of the order of $\pm 12 \%$. The ratio of the neutrino flux-integrated differential CCQElike $\left(d \sigma / d Q^{2}\right)_{\mathrm{QE}+\mathrm{MEC}}$ cross section to the genuine $\mathrm{QE}$ $\left(d \sigma / d Q^{2}\right)_{\mathrm{QE}}$ one, $R_{\mathrm{MEC}}=\left(d \sigma / d Q^{2}\right)_{\mathrm{QE}+\mathrm{MEC}} /\left(d \sigma / d Q^{2}\right)_{\mathrm{QE}}$,

FIG. 17. Same as Fig. 16, but for muon kinetic energy bins: $T(\mathrm{GeV})=(1.6-1.8),(1.8-2.0),(2.0-2.2)$, and (2.2-2.4).
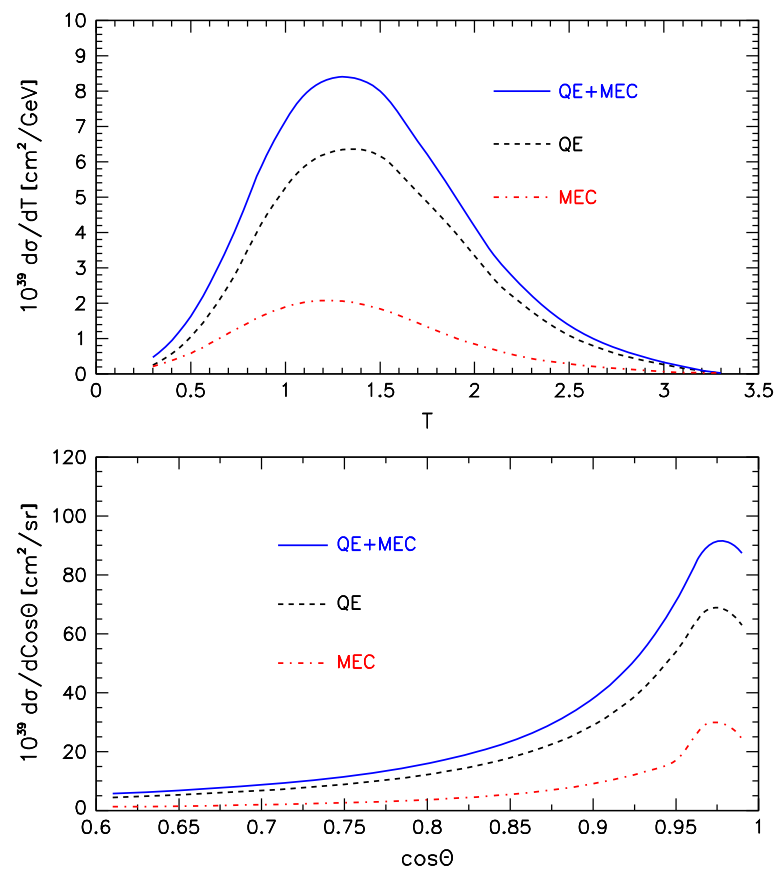

FIG. 18. NOvA flux-integrated $d \sigma / d T$ cross sections for $0.6<$ $\cos \theta<1$ as a function of muon kinetic energy (upper panel) and $d \sigma / d \cos \theta$ cross section for $0.2<T<3.5 \mathrm{GeV}$ as a function of muon scattering angle (lower panel) for the $\nu_{\mu}$ CCQE-like scattering per target neutron. As shown in the key, the cross sections were calculated within the RDWIA + MEC approach. The CCQE and $2 p-2 h$ MEC contributions are also shown. 


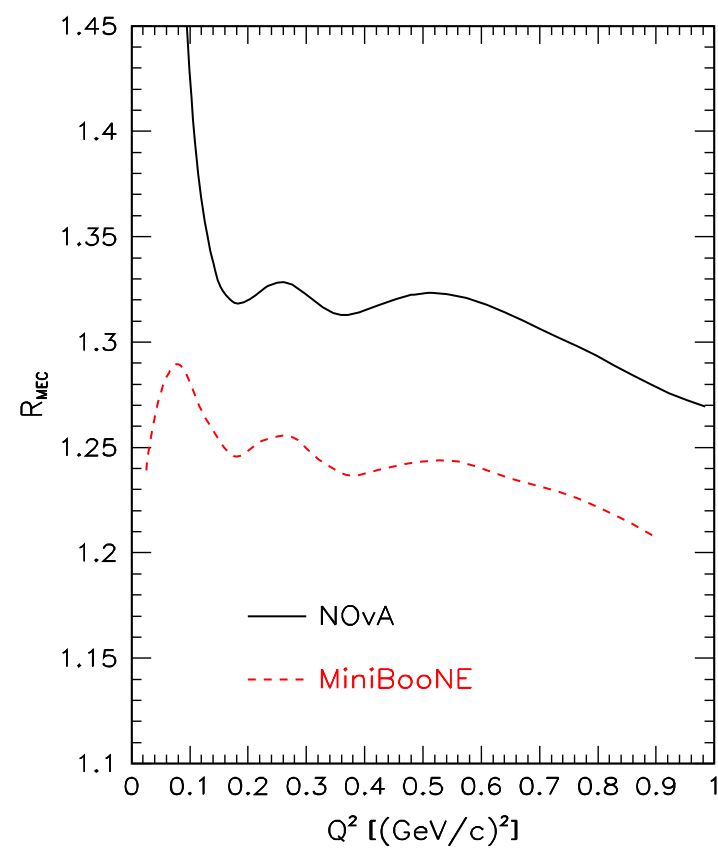

FIG. 19. The ratio $R_{\mathrm{MEC}}$ as a function of $Q^{2}$ for the NOvA and MiniBooNE flux-integrated $d \sigma / d Q^{2}$ cross sections.

calculated for the MiniBooNE and NOvA experiments, is shown in Fig. 19. As observed, in the NOvA case, the contribution of the $2 p-2 h$ MEC is about $8 \%$ higher than in the MiniBooNE experiment. This can be connected with the NOvA neutrino flux that is centered at neutrino energy $\approx 2 \mathrm{GeV}$, whereas the MiniBooNE neutrino flux has maximum at $\approx 0.7 \mathrm{GeV}$.

\section{CONCLUSIONS}

In this article, we analyzed the MiniBooNE neutrino data within the RDWIA + MEC model. This model has been validated in the vector sector by describing the set of inclusive electron scattering ${ }^{12} \mathrm{C}$ data. We performed a shape-only fit of the RDWIA + MEC approach to the data with only the nucleon axial mass, as variable model parameter. A best-fit value of $M_{A} \approx 1.20 \mathrm{GeV}$ was obtained. This value is in agreement within the errors with the best-fit value of $M_{A}=1.15 \pm 0.03 \mathrm{GeV}$ obtained from the CCQE main fit of the MiniBooNE and MINERvA data $[48,62]$. We also extracted the values of the axial form factor $F_{A}\left(Q^{2}\right)$ as a function of $Q^{2}$, using the measured neutrino flux-integrated $d \sigma / d Q^{2}$ cross section. There is good overall agreement within the experimental uncertainties between the extracted values of $F_{A}\left(Q^{2}\right)$ and the dipole parametrization with the value of $M_{A}=1.20 \mathrm{GeV}$. We obtained that in the MiniBooNE experiment the $2 p-2 h$ channel is large, contributing about $25 \%$ depending on kinematics, and it is essential for describing a great amount of experimental data. One can also notice that our calculations are in agreement with other theoretical results which are compatible with MiniBooNE data.

Using the RDWIA + MEC model with $M_{A}=1.20 \mathrm{GeV}$, we estimated the differential flux-integrated CCQE-like cross sections for neutrino scattering in the NOvA near detector. We showed that these cross sections are coincident with ones for neutrino scattering on carbon. The $2 p-2 h$ MEC contributions in the NOvA energy range are about of $30 \%-35 \%$, i.e., approximately $8 \%$ larger than in the MiniBooNE experiment. So, the measurements of the CCQE-like differential cross sections in the NOvA experiment are necessary in order to make precision determinations of neutrino oscillation parameters.

\section{ACKNOWLEDGMENTS}

We greatly acknowledge J. Amaro and G. Megias for fruitful discussions and for putting at our disposal the codes for calculation of the MEC's electroweak response functions that were used in this work. We specially thank A. Habig and J. Samoilova for a critical reading of the manuscript.
[1] R. A. Smith and E. J. Moniz, Nucl. Phys. B43, 605 (1972).

[2] V. Bernard, L. E. Elouadrhiri, and U.-G. Meissner, J. Phys. G 28, R1 (2002).

[3] V. Lyubushkin et al. (NOMAD Collaboration), Eur. Phys. J. C 63, 355 (2009).

[4] G. A. Fiorentini et al. (MINERvA Collaboration), Phys. Rev. Lett. 111, 022502 (2013).

[5] L. Fields et al. (MINERvA Collaboration), Phys. Rev. Lett. 111, 022501 (2013).

[6] L. Aliaga et al. (MINERvA Collaboration), Phys. Rev. D 94, 092005 (2016); 95, 039903(A) (2017).
[7] M. Betancourt, J. Phys. Soc. Jpn. Conf. Proc. 12, 010016 (2016).

[8] C. E. Patrick et al. (MINERvA Collaboration), Phys. Rev. D 97, 052002 (2018).

[9] A. A. Aguilar-Arevalo et al. (MiniBooNE Collaboration), Phys. Rev. D 81, 092005 (2010).

[10] A. A. Aguilar-Arevalo et al. (MiniBooNE Collaboration), Phys. Rev. D 82, 092005 (2010).

[11] A. A. Aguilar-Arevalo et al. (MiniBooNE Collaboration) Phys. Rev. D 88, 032001 (2013).

[12] R. Gran et al. (K2K Collaboration), Phys. Rev. D 74, 052002 (2006). 
[13] X. Espinal and F. Sanchez, AIP Conf. Proc. 967, 117 (2007).

[14] P. Adamson et al. (MINOS Collaboration), Phys. Rev. D 91, 012005 (2015).

[15] K. Abe et al. (T2K Collaboration), Phys. Rev. D 92, 112003 (2015).

[16] W. M. Alberico, Magda Ericson, and A. Molinari, Ann. Phys. (N.Y.) 154, 356 (1984).

[17] G. T. Garvey, D. A. Harris, H. A. Tanaka, R. Tayloe, and G. P. Zeller, Phys. Rep. 580, 1 (2015).

[18] T. Katori and M. Martini, J. Phys. G 45, 013001 (2018).

[19] L. Alvarez-Ruso et al., Prog. Part. Nucl. Phys. 100, 1 (2018).

[20] C. Maieron, M. C. Martinez, J. A. Caballero, and J. M. Udias, Phys. Rev. C 68, 048501 (2003).

[21] M. C. Martinez, P. Lava, N. Jachowicz, J. Ryckebusch, K. Vantournhout, and J. M. Udias, Phys. Rev. C 73, 024607 (2006).

[22] A. Meucci, C. Giusti, and F. D. Pacati, Nucl. Phys. A739, 277 (2004).

[23] A. V. Butkevich and S. A. Kulagin, Phys. Rev. C 76, 045502 (2007).

[24] A. V. Butkevich, Phys. Rev. C 80, 014610 (2009).

[25] A. V. Butkevich and D. Perevalov, Phys. Rev. C 84, 015501 (2011).

[26] M. Martini, M. Ericson, G. Chanfray, and J. Marteau, Phys. Rev. C 81, 045502 (2010).

[27] N. Jachowicz, K. Heyde, J. Ryckebusch, and S. Rombouts, Phys. Rev. C 65, 025501 (2002).

[28] J. Nieves, J. E. Amaro, and M. Valverde, Phys. Rev. C 70, 055503 (2004).

[29] O. Benhar, N. Farina, H. Nakamura, M. Sakuda, and R. Seki, Phys. Rev. D 72, 053005 (2005).

[30] R. Gonzalez-Jimenez, G. D. Megias, M. B. Barbaro, J. A. Caballero, and T. W. Donnelly, Phys. Rev. C 90, 035501 (2014).

[31] T. Leitner, L. Alvarez-Ruso, and U. Mosel, Phys. Rev. C 73, 065502 (2006).

[32] A. Bodek, H. Budd, and M. Christy, Eur. Phys. J. C 71, 1726 (2011).

[33] V. Pandey, N. Jachowicz, J. Ryckebusch, T. Van Cuyck, and W. Cosyn, Phys. Rev. C 89, 024601 (2014).

[34] K. Gallmeister, U. Mosel, and J. Weil, Phys. Rev. C 94, 035502 (2016).

[35] M. Martini, M. Ericson, and G. Chanfray, Phys. Rev. C 84, 055502 (2011).

[36] M. Martini and M. Ericson, Phys. Rev. C 87, 065501 (2013).

[37] J. Nieves, I. Ruiz Simo, and M. J. Vicente Vacas, Phys. Lett. B 707, 72 (2012).

[38] J. Nieves, I. Ruiz Simo, and M. J. Vicente Vacas, Phys. Lett. B 721, 90 (2013).
[39] A. De Pace, M. Nardi, W. M. Alberico, T. W. Donnelly, and A. Molinari, Nucl. Phys. A726, 303 (2003).

[40] I. Ruiz Simo, J. E. Amaro, M. B. Barbaro, A. De Pace, J. A. Caballero, and T.W. Donnelly, J. Phys. G 44, 065105 (2017).

[41] G. D. Megias, T. W. Donnelly, O. Moreno, C. F. Williamson, J. A. Caballero, R. Gonzalez-Jimenez, A. De Pace, M. B. Barbaro, W. M. Alberico, M. Nardi, and J. E. Amaro, Phys. Rev. D 91, 073004 (2015).

[42] G. D. Megias, J. E. Amaro, M. B. Barbaro, J. A. Caballero, T. W. Donnelly, and I. R. Simo, Phys. Rev. D 94, 093004 (2016).

[43] Noemi Rocco, Carlo Barbieri, Omar Benhar, Arturo De Pace, and Alessandro Lovato, Phys. Rev. C 99, 025502 (2019).

[44] A. V. Butkevich and S. V. Luchuk, Phys. Rev. C 97, 045502 (2018).

[45] J. J. Kelly, Adv. Nucl. Phys. 23, 75 (1996).

[46] K. G. Fissum et al., Phys. Rev. C 70, 034606 (2004).

[47] J. J. Kelly, Phys. Rev. C 71, 064610 (2005).

[48] C. Wilkinson et al., Phys. Rev. D 93, 072010 (2016).

[49] A. V. Butkevich and D. Perevalov, Phys. Rev. D 89, 053014 (2014).

[50] A. V. Butkevich, Phys. Rev. C 82, 055501 (2010).

[51] D. S. Ayres et al. (NOvA Collaboration), NOvA Technical Design Report No. FERMILAB-DESIGN-2007-01.

[52] P. Adamson et al. (NOvA Collaboration), Phys. Rev. Lett. 116, 151806 (2016).

[53] P. Mergell, U.-G. Meissner, and D. Drechsel, Nucl. Phys. A596, 367 (1996).

[54] T. de Forest, Nucl. Phys. A392, 232 (1983).

[55] B. Serot and J. Walecka, Adv. Nucl. Phys. 16, 1 (1986).

[56] C. J. Horowitz, D. P. Murdock, and Brian D. Serot, in Computational Nuclear Physics 1: Nuclear Structure, edited by K. Langanke, J. A. Maruhn, and Steven E. Koonin (Springer-Verlag, Berlin, 1991), p. 129.

[57] D. Dutta et al., Phys. Rev. C 68, 064603 (2003).

[58] E. D. Cooper, S. Hama, B. C. Clark, and R. L. Mercer, Phys. Rev. C 47, 297 (1993).

[59] C. Ciofi degli Atti and S. Simula, Phys. Rev. C 53, 1689 (1996).

[60] G. D. Megias and J. E. Amaro (private communication).

[61] G. D. Megias, J. E. Amaro, M. B. Barbaro, J. A. Caballero, and T. W. Donnelly, Phys. Rev. D 94, 013012 (2016).

[62] C. Wilkinson, Constraining neutrino interaction uncertainties for oscillation experiments, Ph.D. thesis, University of Sheffild, 2015.

[63] L. Aliaga, Ph.D. thesis, College of William and Mary, 2016.

[64] X. Bu, arXiv:1601.01213.

[65] A. V. Butkevich, Phys. Rev. C 85, 065501 (2012).

[66] G. D. Megias, M. B. Barbaro, J. A. Caballero, J. E. Amaro, T. W. Donnelly, I. Ruiz Simo, and J. W. Van Orden, J. Phys. G 46, 015104 (2019). 